\title{
Semistability and simple connectivity at $\infty$ of finitely generated groups with a finite series of commensurated subgroups
}

\author{
MiCHAEL L MiHALIK
}

\begin{abstract}
A subgroup $H$ of a group $G$ is commensurated in $G$ if for each $g \in G, g \mathrm{Hg}^{-1} \cap H$ has finite index in both $H$ and $g H^{-1}$. If there is a sequence of subgroups $H=$ $Q_{0} \prec Q_{1} \prec \cdots \prec Q_{k} \prec Q_{k+1}=G$ where $Q_{i}$ is commensurated in $Q_{i+1}$ for all $i$, then $Q_{0}$ is subcommensurated in $G$. In this paper we introduce the notion of the simple connectivity at $\infty$ of a finitely generated group (in analogy with that for finitely presented groups). Our main result is this: if a finitely generated group $G$ contains an infinite finitely generated subcommensurated subgroup $H$ of infinite index in $G$, then $G$ is one-ended and semistable at $\infty$. If, additionally, $G$ is recursively presented and $H$ is finitely presented and one-ended, then $G$ is simply connected at $\infty$. A normal subgroup of a group is commensurated, so this result is a strict generalization of a number of results, including the main theorems in works of G Conner and M Mihalik, B Jackson, V M Lew, M Mihalik, and J Profio. We also show that Grigorchuk's group (a finitely generated infinite torsion group) and a finitely presented ascending HNN extension of this group are simply connected at $\infty$, generalizing the main result of a paper of L Funar and DE Otera.
\end{abstract}

20F65; 20F69, 57M10

\section{Introduction and background}

In 1962, J Stallings defined what it means for a space to be $n$-connected at $\infty$, and proved the following:

Theorem 1.1 (Stallings [23]) If $V^{n}, n \geq 5$, is a contractible PL $n$-manifold without boundary, then $V$ is PL homeomorphic to $R^{n}$ if and only if $V$ is simply connected at $\infty$.

In 1974, R Lee and F Raymond first considered the fundamental group of an end of a group. In particular, they considered groups that are simply connected at $\infty$. 
Theorem 1.2 (Lee and Raymond [14]) Let $G$ be a finitely presented group with normal subgroup $N$ isomorphic to $\mathbb{Z}^{k}$ and quotient $K=G / N$. Assume when $k=1$ that $K$ is one-ended and that when $k=2$ that $K$ is not finite, and no restrictions when $k>2$. Then $G$ is simply connected at $\infty$.

For a reasonable space $X$ (or finitely presented group $G$ ), one needs to know that $X$ (respectively $G$ ) is semistable at $\infty$ in order to have the fundamental group of an end of $X$ (respectively $G$ ) defined independent of base ray. In 1982, B Jackson generalized Theorem 1.2 and in 1983, M Mihalik proved the first semistability at $\infty$ theorem for a class of finitely presented groups. These two results serve as a starting point for this paper.

Theorem 1.3 (Jackson [11]) If $H$ is an infinite finitely presented normal subgroup of infinite index in the finitely presented group $G$, and either $H$ or $G / H$ is one-ended, then $G$ is simply connected at $\infty$.

Theorem 1.4 (Mihalik [16]) If $H$ is an infinite finitely generated normal subgroup of infinite index in the finitely presented group $G$, then $G$ is semistable at $\infty$.

In 1985, the following connections were drawn between semistability and simple connectivity at $\infty$, and group cohomology.

Theorem 1.5 (Geoghegan and Mihalik [6]) If $G$ is a finitely presented and semistable at $\infty$ group then $H^{2}(G, \mathbb{Z} G)$ is free abelian. If $G$ is simply connected at $\infty$ then $H^{2}(G, \mathbb{Z} G)=0$.

It is unknown whether or not all finitely presented groups are semistable at $\infty$. It is also unknown whether or not for all finitely presented groups $G, H^{2}(G, \mathbb{Z} G)$ is free abelian. The main theorem in the unpublished $1993 \mathrm{PhD}$ dissertation of V Ming Lew generalized Theorem 1.4 and the main theorem of the $1990 \mathrm{PhD}$ dissertation of J Profio generalized Theorem 1.3.

Theorem 1.6 (Lew [15]) Suppose $H$ is an infinite finitely generated subnormal subgroup of the finitely generated group $G$, then

$$
H=N_{0} \triangleleft N_{1} \triangleleft N_{2} \triangleleft \cdots \triangleleft N_{k}=G
$$

for $k \geq 1$, and $H$ has infinite index in $G$. Then $G$ is one-ended and semistable at $\infty$.

Theorem 1.7 (Profio [21]) Suppose $H \triangleleft N \triangleleft G$ is a normal series with $H$ and $G$ finitely presented, and $H$ one-ended and of infinite index in $G$. Then $G$ is simply connected at $\infty$. 
Given a subgroup $H$ of a group $G$, the element $g \in G$ is in the commensurator of $H$ in $G$ (denoted $\operatorname{Comm}(H, G)$ ) if $g_{H g}^{-1} \cap H$ has finite index in both $H$ and $g H^{-1}$. The subgroup $H$ is commensurated in $G$ if $\operatorname{Comm}(H, G)=G$, so normal subgroups are commensurated. The main result of G Conner and Mihalik [3] generalizes Theorems 1.4 and 1.3 in a direction different than these last two results.

Theorem 1.8 (Conner and Mihalik [3]) If a finitely generated group $G$ has an infinite finitely generated commensurated subgroup $Q$, and $Q$ has infinite index in $G$, then $G$ is one-ended and semistable at $\infty$. Furthermore, if $G$ and $Q$ are finitely presented and either $Q$ is one-ended or the pair $(G, Q)$ has one filtered end, then $G$ is simply connected at $\infty$.

Example For $p$ a prime, the group $\mathrm{SL}_{n}\left(\mathbb{Z}\left[\frac{1}{p}\right]\right)$ is finitely presented. When $n>2$, the only normal subgroups of this group are either finite or of finite index; see [22]. For $n>2$, the finitely presented one-ended subgroup $\mathrm{SL}_{n}(\mathbb{Z})$ is commensurated in $\mathrm{SL}_{n}\left(\mathbb{Z}\left[\frac{1}{p}\right]\right)$ and so by Theorem $1.8, \mathrm{SL}_{n}\left(\mathbb{Z}\left[\frac{1}{p}\right]\right)$ is one-ended and simply connected at $\infty$.

While Lew's theorem improves Theorem 1.4 by replacing normality by subnormality, Profio's result is the best attempt in the last 30 years to improve the normality hypothesis of Theorem 1.3 to subnormality. As a corollary of our main theorem, we obtain the subnormal version of Jackson's Theorem 1.3. The semistability part of Theorem 1.9 is proved first and then used in an essential way in the proof of the simply connected at $\infty$ part of Theorem 1.9. A new idea, the simple connectivity at $\infty$ of a finitely generated group, is introduced and used in a fundamental way to prove the second part of Theorem 1.9. We point out that we cannot prove this part of Theorem 1.9, even in the finitely presented case, without this new concept.

If $Q$ is a commensurated subgroup of $G$ we use the notation $Q \prec G$. The main theorem of this article is the following.

Theorem 1.9 (main theorem) Suppose $H$ is a finitely generated infinite subgroup of infinite index in the finitely generated group $G$, and $H$ is subcommensurated in $G$ :

$$
H=Q_{0} \prec Q_{1} \prec \cdots \prec Q_{k} \prec G .
$$

Then $G$ is one-ended and semistable at $\infty$. If, additionally, $H$ is one-ended and finitely presented and $G$ is finitely generated and recursively presented, then $G$ is simply connected at $\infty$. 
In the next section we define what it means for a finitely generated (and recursively presented) group to be simply connected at $\infty$ (a strict generalization of simple connectivity at $\infty$ for finitely presented groups).

Example In Mihalik [20], short exact sequences are produced for each $n>0$ of the form

$$
1 \rightarrow H \rightarrow\left(\mathbb{Z}^{n} * \mathbb{Z}\right) \times\left(\mathbb{Z}^{n} * \mathbb{Z}\right) \rightarrow \mathbb{Z}^{n} \rightarrow 1,
$$

where $H$ is one-ended and finitely generated. The group $\mathbb{Z}^{n}$ is $(n-2)$-connected at $\infty$, yet $\left(\mathbb{Z}^{n} * \mathbb{Z}\right) \times\left(\mathbb{Z}^{n} * \mathbb{Z}\right)$ is not simply connected at $\infty$. These examples show that the finitely presented hypothesis on $H$ in Theorems 1.3 and 1.9, cannot be easily relaxed.

R Grigorchuk constructed a finitely generated infinite torsion group $G$ and a finitely presented HNN extension $H$ of $G$; see Grigorchuk [7; 8]. The group $G$ contains a subgroup of finite index $T$, and $T$ is isomorphic to $T \times T$; see de la Harpe [9, Chapter VIII, Theorem 28]. We show that a recursively presented direct product of a one-ended finitely generated group with an infinite finitely generated group is simply connected at $\infty$, and so Grigorchuk's group $G$ is simply connected at $\infty$. We also show that an ascending HNN extension of a one-ended finitely generated recursively presented semistable at $\infty$ and simply connected at $\infty$ group is simply connected at $\infty$. This implies that the HNN extension of Grigorchuck's group is simply connected at $\infty$.

The remainder of the paper is organized as follows. In Section 2, the working definitions and notation are established. We introduce our definition of a subgroup being simply connected at $\infty$ inside an overgroup. This definition is then used to define the simple connectivity at $\infty$ of a finitely generated group. We end Section 2 with a few important technical lemmas.

In Section 3, we prove the semistability part of our main theorem. This is an induction argument that starts with base case given by Theorem 1.8.

In Section 4, we prove the simple connectivity at $\infty$ part of our main theorem. This is also an induction argument that starts with the base case given by the simple connectivity part of Theorem 1.8. The semistability result of Section 3 is used in conjunction with Lemma 2.9 to set up the proof of the simple connectivity part of Theorem 1.9.

Finally, in Section 5 we prove two simply connected at $\infty$ results, and apply the results to show Grigorchuk's group and its HNN extension are simply connected at $\infty$. 


\section{Definitions and a technical lemma}

Geoghegan's book [5] is a general reference to all that is in this section. A continuous function $f: X \rightarrow Y$ is proper if for each compact subset $C$ of $Y, f^{-1}(C)$ is compact in $X$. A proper map $r:[0, \infty) \rightarrow X$ is called a ray in $X$. If $K$ is a locally finite, connected $\mathrm{CW}$-complex, then one can define an equivalence relation $\sim$ on the set $A$ of all rays in $K$ by setting $r \sim s$ if and only if for each compact set $C \subset K$, there exists an integer $N(C)$ such that $r([N(C), \infty))$ and $s([N(C), \infty))$ are contained in the same unbounded path component of $K-C$ (a path component of $K-C$ is unbounded if it is not contained in any compact subset of $K$ ). An equivalence class of $A / \sim$ is called an end of $K$, the set of equivalence classes of $A / \sim$ is called the set of ends of $K$, and two rays in $K$ in the same equivalence class are said to converge to the same end. The cardinality of $A / \sim$, denoted by $e(K)$, is the number of ends of $K$.

If $G$ is a finitely generated group with generating set $\mathcal{S}$, then the Cayley graph of $G$ with respect to $\mathcal{S}$, denoted $\Gamma_{(G, \mathcal{S})}$, has vertex set $G$ and an edge between vertices $v$ and $w$ if $v s=w$ for some $s \in \mathcal{S}$. We define the number of ends of $G$, denoted by $e(G)$, to be the number of ends of the Cayley graph of $G$ with respect to a finite generating set. (In particular, $e(G)=e\left(\Gamma_{(G, \mathcal{S})}\right.$.) This definition is independent of the choice of finite generating set for $G$. If $G$ is finitely generated, then $e(G)$ is either $0,1,2$, or is infinite (in which case it has the cardinality of the real numbers). We let $*$ denote the base point of $\Gamma_{(G, \mathcal{S})}$, which corresponds to the identity of $G$.

If $f$ and $g$ are rays in $K$, then one says that $f$ and $g$ are properly homotopic if there is a proper map $H:[0,1] \times[0, \infty) \rightarrow K$ such that $\left.H\right|_{\{0\} \times[0, \infty)}=f$ and $\left.H\right|_{\{1\} \times[0, \infty)}=g$. If $f(0)=g(0)=v$ and $\left.H\right|_{[0,1] \times\{0\}}=v$, one says $f$ and $g$ are properly homotopic relative to $v$ (or $\operatorname{rel}\{v\}$ ).

Definition 2.1 A locally finite connected CW-complex $K$ is semistable at $\infty$ if any two rays in $K$ converging to the same end are properly homotopic. The space $K$ is simply connected at $\infty$ if for any compact set $C \subset K$ there is a compact $D \subset K$ such that loops in $K-D$ are homotopically trivial in $K-C$.

In a locally finite $\mathrm{CW}$-complex, any ray is properly homotopic to an edge path ray. So in order to show semistability in such a complex, it is enough to prove edge path rays converging to the same end are properly homotopic.

[16, Theorem 2.1] and [18, Lemma 9] provide several equivalent notions of semistability. The space considered in [16] is simply connected, but simple connectivity is not important in that argument. A slight modification of proofs gives the following result; see [3]. 
Theorem 2.2 Suppose $K$ is a locally finite connected one-ended $C W$-complex. Then the following are equivalent:

(1) $K$ is semistable at $\infty$.

(2) For any ray $r:[0, \infty) \rightarrow K$ and compact set $C$, there is a compact set $D$ such that for any third compact set $E$ and loop $\alpha$ based on $r$ and with image in $K-D, \alpha$ is homotopic rel $\{r\}$ to a loop in $K-E$, by a homotopy with image in $K-C$.

(3) For some (equivalently any) ray $r$ in $K$ and any collection of compact sets $C_{i}$ such that $\bigcup_{i=1}^{\infty} C_{i}=K$ and $C_{i-1}$ is a subset of the interior of $C_{i}$, the inverse system

$$
\pi_{1}\left(X-C_{1}, r\right) \leftarrow \pi_{1}\left(X-C_{2}, r\right) \leftarrow \cdots
$$

with bonding maps induced by inclusion along $r$, is proisomorphic to an inverse system of groups with epimorphic bonding maps.

(4) For any compact set $C$ there is a compact set $D$ such that if $r$ and $s$ are rays based at $v$ and with image in $K-D$, then $r$ and $s$ are properly homotopic $\operatorname{rel}\{v\}$ by a proper homotopy in $K-C$.

If $K$ is simply connected (or if a group acting by homeomorphisms on $K$, acts transitively on the vertices of $K$ ) then a fifth equivalent condition can be added to this list:

(5) If $r$ and $s$ are rays based at $v$, then $r$ and $s$ are properly homotopic $\operatorname{rel}\{v\}$.

If finite connected $\mathrm{CW}$-complexes $X$ and $Y$ have isomorphic fundamental groups, then the universal cover of $X$ is semistable (simply connected) at $\infty$ if and only if the universal cover of $Y$ is semistable (simply connected) at $\infty$. This result can be seen from the early work of F E A Johnson [12; 13], or the proof of [14, Theorem 3]. For a complete argument see the first three sections of [5, Chapter 5].

Definition 2.3 If $G$ is a one-ended, finitely presented group and $X$ is some (equivalently any) finite $\mathrm{CW}$-complex with fundamental group $G$, then we say $G$ is semistable at $\infty$ if the universal cover of $X$ is semistable at $\infty$. We say $G$ is simply connected at $\infty$ if the universal cover of $X$ is simply connected at $\infty$.

The notion of semistabilty for a finitely generated group was first defined in [19]. We give the definition for one-ended groups since this is the case that concerns us. Suppose $G$ is a one-ended finitely generated group with generating set $\mathcal{S}:=\left\{g_{1}, g_{2}, \ldots, g_{n}\right\}$ and let $\Gamma_{(G, \mathcal{S})}$ be the Cayley graph of $G$ with respect to this generating set. Suppose $\left\{\alpha_{1}, \alpha_{2}, \ldots, \alpha_{m}\right\}$ is a finite set of relations in $G$ written in the letters $\left\{g_{1}^{ \pm}, g_{2}^{ \pm}, \ldots, g_{n}^{ \pm}\right\}$. 
For any vertex $v \in \Gamma_{(G, \mathcal{S})}$, there is an edge path cycle labeled $\alpha_{i}$ at $v$. The 2dimensional CW-complex $\Gamma_{(G, \mathcal{S})}\left(\alpha_{1}, \ldots, \alpha_{m}\right)$ is obtained by attaching, at each vertex of $\Gamma_{(G, \mathcal{S})}, 2$-cells corresponding to the relations $\alpha_{1}, \ldots, \alpha_{n}$.

We show in [19] that if $\mathcal{S}$ and $\mathcal{T}$ are finite generating sets for the group $G$ and there are finitely many $\mathcal{S}$-relations $P$ such that $\Gamma_{(G, \mathcal{S})}(P)$ is semistable at $\infty$, then there are finitely many $\mathcal{T}$-relations $Q$ such that $\Gamma_{(G, \mathcal{T})}(Q)$ is semistable at $\infty$; hence the following definition.

Definition 2.4 A finitely generated group $G$ is semistable at $\infty$ if for some (equivalently any) finite generating set $\mathcal{S}$ for $G$ and finite set of $\mathcal{S}$-relations $P$ the complex $\Gamma_{(G, \mathcal{S})}(P)$ is semistable at $\infty$.

Note that if $G$ has finite presentation $\langle\mathcal{S}: P\rangle$, then $G$ is semistable at $\infty$ with respect to Definition 2.3 if and only if $G$ is semistable at $\infty$ with respect to Definition 2.4 if and only if $\Gamma_{(G, \mathcal{S})}(P)$ is semistable at $\infty$.

The following definition defines what it means for a finitely generated subgroup of a finitely presented group to be simply connected at $\infty$ relative to the finitely presented overgroup.

Definition 2.5 A finitely generated subgroup $A$ of a finitely presented group $G$ is simply connected at $\infty$ in $G$ (or relative to $G$ ) if for some (equivalently any by Lemma 2.9 with $N=0$ ) finite presentation $\langle\mathcal{A}, \mathcal{B}: R\rangle$ of the group $G$ (where $\mathcal{A}$ generates $A$ and $\mathcal{A} \cup \mathcal{B}$ generates $G)$, the 2-complex $\Gamma_{(G, \mathcal{A} \cup \mathcal{B})}(R)$ has the following property: given any compact set $C \subset \Gamma_{(G, \mathcal{A} \cup \mathcal{B})}(R)$, there is a compact set $D \subset$ $\Gamma_{(G, \mathcal{A} \cup \mathcal{B})}(R)$ such that any edge path loop in $\Gamma_{(A, \mathcal{A})}-D$ is homotopically trivial in $\Gamma_{(G, \mathcal{A} \cup \mathcal{B})}(R)-C$.

In order to define what it means for a finitely generated group $G$ to be simply connected at $\infty$, we must know that $G$ embeds in some finitely presented group. In 1961, $\mathrm{G}$ Higman proved the following.

Theorem 2.6 (Higman [10]) A finitely generated infinite group $G$ can be embedded in a finitely presented group if and only if $G$ is recursively presented.

Definition 2.7 A finitely generated and recursively presented group $A$ is simply connected at $\infty$ if for any finitely presented group $G$ and subgroup $A^{\prime}$ isomorphic to $A$, $A^{\prime}$ is simply connected at $\infty$ in $G$. 
Suppose that $A$ is a finitely presented group and $A$ satisfies the simply connected at $\infty$ condition of Definition 2.3, then $A$ satisfies Definition 2.7. If $A$ is a finitely presented group satisfying Definition 2.7, then if we let $A=G$ in Definition 2.7, we see that $A$ satisfies Definition 2.3, and there is no ambiguity.

We conclude this section with Lemmas 2.9 and 2.10, but first some terminology. Suppose $\langle\mathcal{S}: R\rangle$ is a finite presentation for a group $G$. If $A$ is a subcomplex of $\Gamma_{(G, \mathcal{S})}(R)$, then $\operatorname{St}(A)$ is the subcomplex of $\Gamma_{(G, \mathcal{S})}(R)$ whose vertices $V(\operatorname{St}(A))$ are the vertices of $A$ along with each vertex of $\Gamma_{(G, \mathcal{S})}(R)$ that is connected to a vertex of $A$ by an edge. The edges $E(\operatorname{St}(A))$ of $\operatorname{St}(A)$ are all edges of $A$ union all edges of $\Gamma_{(G, \mathcal{S})}(R)$, both of whose vertices are contained in $V(\operatorname{St}(A))$. The $2-$ cells $F(\operatorname{St}(A))$ of $\operatorname{St}(A)$ are all 2-cells of $A$ union all 2-cells $F$ such that all vertices of $F$ belong to $V(\operatorname{St}(A))$. If $A$ is an arbitrary subset of $\Gamma_{(G, \mathcal{S})}(R)$ then let $\hat{A}$ be the smallest subcomplex of $\Gamma_{(G, \mathcal{S})}(R)$ containing $A$ and define $\operatorname{St}(A)$ to be $\operatorname{St}(\hat{A})$. Inductively, $\mathrm{St}^{L}(A)=\mathrm{St}\left(\mathrm{St}^{L-1}(A)\right)$ for $L>1$.

Lemma 2.8 (1) Suppose $A$ and $B$ are subcomplexes of $\Gamma_{(G, \mathcal{S})}(R)$ and $\operatorname{St}(A) \cap$ $B \neq \varnothing$. Then $A \cap \operatorname{St}(B) \neq \varnothing$.

(2) Suppose $A$ is a subcomplex of $\Gamma_{(G, \mathcal{S})}(R)$ and $B$ is an arbitrary subset of $\Gamma_{(G, \mathcal{S})}(R)$ and $\operatorname{St}(B) \cap A \neq \varnothing$ then $\operatorname{St}^{L+1}(A) \cap B \neq \varnothing$, where $L$ is the length of the longest relation in $R$.

Proof Case (1) If $\operatorname{St}(A) \cap B \neq \varnothing$ then there is a vertex $v \in \operatorname{St}(A) \cap B$. If $v$ is in $A$ then we are finished. Otherwise, there is a vertex $w \in A$ and an edge from $v$ to $w$. Then $w \in A \cap \operatorname{St}(B)$.

Case (2) Let $v$ be a vertex in $\operatorname{St}(B) \cap A=\operatorname{St}(\hat{B}) \cap A$. If $v$ is in $B$ we are finished. Otherwise, $v$ is in $\hat{B}$ or $v$ is adjacent to a vertex $w \in \hat{B}$. If $v$ is in $\hat{B}$ then there is an edge $e$ containing a point $b \in B$ and $v$ is a vertex of $e$, or there is a 2-cell $F$ containing a point $b \in B$ and $v$ is a vertex of $F$. In either case, $b$ is $\operatorname{in}^{L} \operatorname{St}^{L}(v)$, so $b$ is in $B \cap \mathrm{St}^{L}(A)$. If $v$ is adjacent to a vertex $w \in \hat{B}$ then as above, there is $b \in B \cap \mathrm{St}^{L}(w) \subset B \cap \mathrm{St}^{L+1}(A)$.

The following technical lemma has a somewhat standard proof.

Lemma 2.9 Suppose $A$ is a finitely generated subgroup of the finitely presented group $G$. Then $A$ is simply connected at $\infty$ in $G$ if and only if the following holds:

$(\dagger)$ For $\langle\mathcal{S}: R\rangle$ an arbitrary finite presentation for $G, N \geq 0$ an integer and $C$ a compact subset of $\Gamma_{(G, \mathcal{S})}(R)$, there is a compact set $D(C, N) \subset \Gamma$ such that if $\alpha$ is an edge path loop in $\Gamma-D$ and each vertex of $\alpha$ is within $N$ of some vertex of $A(\subset \Gamma)$, then $\alpha$ is homotopically trivial in $\Gamma-C$. 
Proof If condition ( $\dagger$ ) holds with $N=0$ then clearly $A$ is simply connected at $\infty$. For the converse, assume $A$ is simply connected at $\infty$ and $\langle\mathcal{A}, \mathcal{B}: T\rangle$ is a presentation for $G$ satisfying the conditions of Definition 2.5. Define $\Gamma_{1}:=\Gamma_{(G, \mathcal{A} \cup \mathcal{B})}(T)$ and $\Gamma_{2}:=\Gamma_{(G, \mathcal{S})}(R)$. Recall that the vertices of $\Gamma_{1}$ and of $\Gamma_{2}$ are both the elements of $G$. In order to avoid confusion if $v$ is a vertex of $\Gamma_{1}$ we denote by $v^{\prime}$ the corresponding vertex of $\Gamma_{2}$. We define proper maps respecting the action of $G, f_{1}: \Gamma_{1} \rightarrow \Gamma_{2}$ and $f_{2}: \Gamma_{2} \rightarrow \Gamma_{1}$ such that for each vertex $g \in G$ of $\Gamma_{1}, f_{1}(g)=g^{\prime}$ and $f_{2}\left(g^{\prime}\right)=g$. If $e$ is an edge of $\Gamma_{1}$ with initial vertex $v$, terminal vertex $w$ and label $s \in \mathcal{A} \cup \mathcal{B}$, then choose an edge path $\tau_{s}$ in $\Gamma_{2}$ from $v^{\prime}:=f_{1}(v)$ to $w^{\prime}:=f_{1}(w)$. Define $f_{1}(e)$ to be $\tau_{s}$. If $g \in G$ define $f_{1}$ on $g e$ to be $g \tau_{s}$. Similarly define $f_{2}$ from the 1-skeleton of $\Gamma_{2}$ to the 1 -skeleton of $\Gamma_{1}$. Let $M_{1}$ be the length of the longest path $\tau_{s}$ for $s \in \mathcal{A} \cup \mathcal{B}$ and $M_{2}$ be the length of the longest path $\tau_{s^{\prime}}$ for $s^{\prime} \in \mathcal{S}$. Note that if $e$ is an edge of $\Gamma_{1}$, with initial vertex $v$ and terminal vertex $w$, then $f_{2} f_{1}(e)$ is an edge path of length at most $M_{1} M_{2}$ from $v$ to $w$, and similarly if $e$ is an edge of $\Gamma_{2}$.

In particular, if $x$ is a point of an edge of $\Gamma_{1}$ then $f_{2}\left(f_{1}(x)\right) \in \mathrm{St}^{M_{1} M_{2}}(x)$, and likewise if $x$ belongs to an edge of $\Gamma_{2}$.

If $F$ is a $2-$ cell of $\Gamma_{1}$, then the boundary of $F$ is an edge path $\beta_{F}$ with edge labels the same as an element of $T$. Then $f_{1}\left(\beta_{F}\right)$ is an edge path loop in $\Gamma_{2}$. Choose $P_{1}>0$ so that if $F$ is any 2 -cell of $\Gamma_{1}$, then the edge path loop $f_{1}\left(\beta_{F}\right)$ is homotopically trivial in $\operatorname{St}^{P_{1}}\left(v^{\prime}\right)$ for any vertex $v^{\prime}$ of $f_{1}\left(\beta_{F}\right)$. The map $f_{1}$ is defined so that $\left.f_{1}\right|_{F}$ (the restriction of $f_{1}$ to any 2-cell $F$ ) realizes this homotopy and respects the action of $G$ on $\Gamma_{1}$ and $\Gamma_{2}$. Similarly map the 2-cells of $\Gamma_{2}$ to $\Gamma_{1}$ and choose $P_{2}$ for $f_{2}$. Let $L$ be the length of the longest relator of $T \cup R$.

If $x$ is a point of a 2-cell $F$ of $\Gamma_{1}$ and $v$ is a vertex of $F$, then $f_{1}(x) \in \operatorname{St}^{P_{1}}\left(f_{1}(v)\right)=$ $\mathrm{St}^{P_{1}}\left(v^{\prime}\right)$. This means there is an edge path $\tau$ in $\Gamma_{2}$ of length at most $P_{1}$ from $v^{\prime}$ to a vertex $w^{\prime}$ and $w^{\prime}$ belongs to an edge $b$ or 2-cell $B$ containing $f_{1}(x)$.

If $w^{\prime}$ belongs to an edge $b$ then $f_{2}\left(f_{1}(x)\right)$ belongs to $f_{2}((\tau, b))$ an edge path of length at most $M_{2}\left(P_{1}+1\right)$ that begins at $v$. In this case $f_{2}\left(f_{1}(x)\right) \in \mathrm{St}^{M_{2}\left(P_{1}+1\right)}(x)$.

Otherwise, $w^{\prime}$ belongs to a $2-$ cell $B$ containing $f_{1}(x)$ and $f_{2}\left(f_{1}(x)\right)$ belongs to $\mathrm{St}^{P_{2}}\left(f_{2}\left(w^{\prime}\right)\right)=\mathrm{St}^{P_{2}}(w)$. Then $f_{2}(\tau)$ is an edge path of length at most $P_{1} M_{2}$ from $v$ to $w$, and $f_{2} f_{1}(x) \in \mathrm{St}^{P_{1} M_{1}+P_{2}}(v)$. As $x \in \mathrm{St}^{L}(v)$, we have $f_{2}\left(f_{1}(x)\right) \in$ $\mathrm{St}^{P_{1} M_{1}+P_{2}+L}(x)$. Combining, we make the following claim.

Claim 1 There is an integer $M$ such that if $x$ is a point of $\Gamma_{1}$ (respectively $\Gamma_{2}$ ), then $f_{2}\left(f_{1}(x)\right) \in \mathrm{St}^{M}(x)$ (respectively $\left.f_{1}\left(f_{2}(x)\right) \in \mathrm{St}^{M}(x)\right)$.

Let $\Gamma_{3}:=\Gamma_{(A, \mathcal{A})}$ be the corresponding subgraph of $\Gamma_{1}$. Then for any compact set $C$ in $\Gamma_{1}$ there is a compact set $D$ in $\Gamma_{1}$ so that any edge path loop in $\Gamma_{3}-D$, is 
homotopically trivial in $\Gamma_{1}-C$. Let $\Gamma_{4}:=f_{1}\left(\Gamma_{3}\right)$. Then $A$ is a subset of the vertices of $\Gamma_{4}$ and we call these vertices the pseudo vertices of $\Gamma_{4}$. For each edge $e$ of $\Gamma_{3}$, $f_{1}(e)$ is an edge path of $\Gamma_{4}$ (connecting two pseudo vertices) that we call a pseudo edge of $\Gamma_{4}$.

Claim 2 Given a compact set $C$ in $\Gamma_{2}$, there is a compact set $D_{1}(C)$ in $\Gamma_{2}$ such that any pseudo edge path loop $\beta$ in $\Gamma_{4}-D_{1}$ is homotopically trivial in $\Gamma_{2}-C$.

Proof Assume $C$ is a compact subcomplex of $\Gamma_{2}$. Then $\mathrm{St}^{M+L}\left(f_{2}(C)\right)$ is a compact subcomplex of $\Gamma_{1}$. As $\Gamma_{1}$ satisfies Definition 2.5, there is a compact subcomplex $E$ of $\Gamma_{1}$ such that any edge path loop in $\Gamma_{3}-E$ is homotopically trivial in $\Gamma_{1}-$ $\mathrm{St}^{M+L}\left(f_{2}(C)\right)$.

Choose $D_{1}$ a compact subcomplex of $\Gamma_{2}$ such that if $w \in G$ is a vertex of $E$ then $f_{2}(w):=w^{\prime} \in D_{1}$. If $\beta^{\prime}$ is a pseudo edge path loop in $\Gamma_{4}-D_{1}$, let $\beta$ be an edge path loop in $\Gamma_{3}$ such that $f_{1}(\beta)=\beta^{\prime}$. Note that no vertex of $\beta$ belongs to $E$ and so $\beta$ avoids $E$. Then there is a homotopy $H$ that kills $\beta$ in $\Gamma_{1}-\mathrm{St}^{M+L}\left(f_{2}(C)\right)$ and $f_{1} H$ kills $\beta^{\prime}$ in $\Gamma_{2}$. It remains to show that the image of $f_{1} H$ avoids $C$. If $\operatorname{im}\left(f_{1} H\right) \cap C \neq \varnothing$, then $\operatorname{im}\left(f_{2} f_{1} H\right) \cap f_{2}(C) \neq \varnothing$. By Claim 1 , $\operatorname{im}\left(f_{2} f_{1} H\right) \subset \mathrm{St}^{M}(\mathrm{im}(H))$ and so $\mathrm{St}^{M}(\mathrm{im}(H)) \cap f_{2}(C) \neq \varnothing$. By Lemma 2.8(1), $\operatorname{St}(\operatorname{im}(H)) \cap \mathrm{St}^{M-1}\left(f_{2}(C)\right) \neq \varnothing$ and by Lemma $2.8(2) \operatorname{im}(H) \cap \mathrm{St}^{M+L}\left(f_{2}(C)\right) \neq \varnothing$. But $\operatorname{im}(H) \cap \mathrm{St}^{M+L}\left(f_{2}(C)\right)=\varnothing$.

Now we complete the proof of Lemma 2.9. Recall, $N \geq 0$ is an arbitrary fixed integer. Choose $N_{1}$ such that if two pseudo vertices of $\Gamma_{4}$ are within $2 N+1$ of one another in $\Gamma_{2}$ then there is a pseudo edge path of $\Gamma_{2}$-length at most $N_{1}$ connecting them. Let $C$ be compact in $\Gamma_{2}$. Choose $N_{2}$ so that if $\tau$ is an edge path loop in $\Gamma_{2}$ of length at most $N_{1}+2 N+1$, then $\tau$ is homotopically trivial in $\operatorname{St}^{N_{2}}\left(w^{\prime}\right)$ for any vertex $w^{\prime}$ of $\tau$. Now suppose $\alpha$ is an edge path loop of $\Gamma_{2}-\mathrm{St}^{N_{2}}\left(D_{1}(C)\right)$ and each vertex of $\alpha$ is within $N$ of $A$ (the pseudo vertices of $\Gamma_{4}$ ). By the definition of $N_{2}, \alpha$ is homotopic to a pseudo edge path loop $\alpha^{\prime}$ in $\Gamma_{4}-D_{1}$ by a homotopy in $\Gamma_{2}-D_{1}$. Since $\alpha^{\prime}$ is homotopically trivial in $\Gamma_{2}-C, \alpha$ is as well.

Remark For spaces (and finitely presented groups), simple connectivity at $\infty$ is stronger than semistability at $\infty$. In fact Theorem 2.2(3) states that a space $K$ is semistable at $\infty$ if and only if pro $-\pi_{1}^{\text {end }}(K)$ is proisomorphic to an inverse system of groups with epimorphic bonding maps. The space $K$ is simply connected at $\infty$ if and only if pro $-\pi_{1}^{\text {end }}(K)$ is protrivial. It is not clear whether or not our definition of simple connectivity at $\infty$ for a finitely generated group implies the group is semistable at $\infty$. 
Interestingly, these two notions can be combined in effective ways (see Lemma 2.10 and Theorem 5.2).

Lemma 2.9 implies the following. Suppose the finitely generated group $A$ is simply connected at $\infty$ in the finitely presented group $G,(\mathcal{S}, R)$ is a finite presentation for $G$, and $v_{1}, \ldots, v_{n}$ are vertices of $\Gamma_{(G, \mathcal{S})}(R)$. Then for any compact $C \subset \Gamma$ and integer $N \geq 0$ there is a compact set $D\left(C, N,\left\{v_{1}, \ldots, v_{n}\right\}\right)$ such that any loop in $\Gamma-D$, each of whose vertices is within $N$ of $v_{i} A$ for some $i \in\{1, \ldots, n\}$, is homotopically trivial in $\Gamma-C$. What is not guaranteed is a compact set $D(C, N)$ satisfying the following: for all $v \in G$ and any edge path loop $\alpha$ in $\Gamma-D$ with each vertex of $\alpha$ within $N$ of $v A$, the loop $\alpha$ is homotopically trivial in $\Gamma-C$. We do gain this enhanced condition (see Lemma 2.10) if $A$ is both simply connected at $\infty$ in $G$ and semistable at $\infty$.

Lemma 2.10 Suppose $A$ is a finitely generated semistable at $\infty$ group and $A$ is simply connected at $\infty$ in the finitely presented group $G$, then the following holds:

(\$) For $\langle\mathcal{S}: R\rangle$ an arbitrary finite presentation for $G, N \geq 0$ an integer and $C$ a compact subcomplex of $\Gamma_{(G, \mathcal{S})}(R)$, there is a compact set $D(C, N) \subset \Gamma$ such that if $\alpha$ is an edge path loop in $\Gamma-D, v$ is an element of $G$, and each vertex of $\alpha$ is within $N$ of the coset $v A(\subset \Gamma)$, then $\alpha$ is homotopically trivial in $\Gamma-C$.

Proof By Lemma 2.9, condition ( $\$$ ) holds for any finite collection of vertices $v$. For the sake of simplicity, we assume that $\mathcal{S}$ contains a set of generators $\mathcal{A}$ for $A$, and $R$ contains a finite set of $\mathcal{A}$-relations $R_{\mathcal{A}}$ so that $\Gamma_{(A, \mathcal{A})}\left(R_{\mathcal{A}}\right)$ is semistable at $\infty$. Choose an integer $M$ so that if $\alpha$ is an edge path loop in $\Gamma_{(G, \mathcal{S})}(R)$ and each vertex of $\alpha$ is within $N$ of $A \subset \Gamma_{(G, \mathcal{S})}(R)$ then $\alpha$ is homotopic to an edge path loop $\alpha^{\prime}$ in $\Gamma_{(A, \mathcal{A})}\left(R_{\mathcal{A}}\right)$ by a homotopy in $\operatorname{St}^{M}(\operatorname{image}(\alpha)) \subset \Gamma_{(G, \mathcal{S})}(R)$. Choose a finite set of vertices $v_{1}, \ldots, v_{n}$ such that if $v \Gamma_{(A, \mathcal{A})}\left(R_{\mathcal{A}}\right) \cap \mathrm{St}^{M}(C) \neq \varnothing$ then $v A=v_{i} A$ for some $i \in\{1, \ldots, n\}$. Choose $D\left(\mathrm{St}^{M}, N,\left\{v_{1}, \ldots, v_{n}\right\}\right)$ compact (as in the remark on page 3624) so that any loop in $\Gamma_{(G, \mathcal{S})}(R)-D$, each of whose vertices is within $N$ of $v_{i} A$ for some $i \in\{1, \ldots, n\}$, is homotopically trivial in $\Gamma_{(G, \mathcal{S})}(R)-C$. If $v A \neq v_{i} A$ for any $i \in\{1, \ldots, n\}$, and $\alpha$ is a loop in $\Gamma_{(G, \mathcal{S})}(R)-D$ such that each vertex of $\alpha$ is within $N$ of $v A$, then by the definition of $M, \alpha$ is homotopic to a loop $\alpha^{\prime}$ in $v \Gamma_{(A, \mathcal{A})}\left(R_{\mathcal{A}}\right)$ by a homotopy avoiding $C$. Since $A$ is simply connected in $G$ there is a compact set $E$ such that any loop in $v \Gamma_{(A, \mathcal{A})}\left(R_{\mathcal{A}}\right)-E$ is homotopically trivial in $\Gamma_{(G, \mathcal{S})}(R)-C$. Since $A$ is semistable at $\infty, \alpha^{\prime}$ is homotopic in $v \Gamma_{(A, \mathcal{A})}\left(R_{\mathcal{A}}\right)$ to a loop $\alpha^{\prime \prime}$ in $v \Gamma_{(A, \mathcal{A})}\left(R_{\mathcal{A}}\right)-E$. Since $C \cap v \Gamma_{(A, \mathcal{A})}\left(R_{\mathcal{A}}\right)=\varnothing$ this homotopy avoids $C$. Now $\alpha^{\prime \prime}$ is homotopically trivial in $\Gamma_{(G, \mathcal{S})}(R)-C$ and so $\alpha$ is also. 


\section{Semistability}

For the remainder of the paper, we assume that $G$ is a finitely generated group, $H$ is an infinite finitely generated subgroup of infinite index in $G$ and (as in the statement of Theorem 1.9) $H$ is subcommensurated in $G$ :

$$
H=Q_{0} \prec Q_{1} \prec \cdots \prec Q_{k} \prec Q_{k+1}=G .
$$

Let $\mathcal{H}:=\left\{h_{1}, \ldots, h_{n}\right\}$ be a finite generating set for $H$, and suppose the group $G$ has generating set $\mathcal{G}:=\left\{h_{1}, \ldots, h_{n}, s_{1}, \ldots, s_{m}\right\}$. Let $\mathcal{S}:=\left\{s_{1}, \ldots, s_{m}\right\}$.

Lemma 3.1 [2, Lemma 3.1] Suppose $Q$ and $B$ are subgroups of the group $G$ and $Q \prec G$. Then $Q \cap B \prec B$.

Lemma 3.2 If $H$ is a subgroup of $A$ and $A$ is a subgroup of $G(H<A<G)$, then

$$
H=Q_{0} \prec Q_{1} \cap A \prec \cdots \prec Q_{k} \cap A \prec A .
$$

Proof Recall $Q_{k+1}:=G$. For $i=1, \ldots, k+1$, define $B_{i}:=A \cap Q_{i}$. As $Q_{i-1} \prec Q_{i}$ and $B_{i}<Q_{i}$, Lemma 3.1 implies that $Q_{i-1} \cap B_{i} \prec B_{i}$. Equivalently, $Q_{i-1} \cap A \prec$ $Q_{i} \cap A$.

Lemma 3.3 Suppose $i \in\{1,2, \ldots, k+1\}, g \in Q_{i}$ and $Y$ is a subgroup of $Q_{i-1}$. Then $g^{-1} Q_{i-1} g \cap Y$ has finite index in $Y$ and so $g Y^{-1} \cap Q_{i-1}$ has finite index in $g Y g^{-1}$. Note that if $Y$ is finitely generated, then $g^{-1} Q_{i-1} g \cap Y$ and $Q_{i-1} \cap g Y g^{-1}$ are as well.

Proof The group $g^{-1} Q_{i-1} g \cap Q_{i-1}$ has finite index in $Q_{i-1}$. Therefore, the group $g^{-1} Q_{i-1} g \cap Q_{i-1} \cap Y=g^{-1} Q_{i-1} g \cap Y$ has finite index in $Y$. Conjugating, we have that the group $Q_{i-1} \cap g Y g^{-1}$ has finite index in $g Y g^{-1}$.

For $s \in \mathcal{S}^{ \pm 1}$ let $\mathcal{A}_{s}$ be a finite generating set for $Q_{k} \cap s^{-1} H s$, and define

$$
\mathcal{A}_{1}:=\bigcup_{s \in \mathcal{S}^{ \pm 1}} \mathcal{A}_{s} \quad \text { and } \quad \mathcal{A}:=\mathcal{H} \cup \mathcal{A}_{1} .
$$

Then $A:=\langle\mathcal{A}\rangle$ is a finitely generated subgroup of $Q_{k}$.

The following two lemmas imply the semistability part of Theorem 1.9.

Lemma 3.4 If $H$ has finite index in $A$, then $H$ is commensurated in $G$ (and so $G$ is one-ended and semistable at $\infty$ by Theorem 1.8). 
Lemma 3.5 If $H$ has infinite index in $A$, then $H$ is subcommensurated in $A$ :

$$
H=Q_{0} \prec Q_{1} \cap A \prec \cdots \prec Q_{k} \cap A=A,
$$

and both $A$ and $G$ are one-ended and semistable at $\infty$.

Proof of Lemma 3.4 It suffices to show that for $s \in \mathcal{S}^{ \pm 1}, s^{-1} H s \cap H$ has finite index in both $H$ and $s^{-1} H s$. Since $H$ has finite index in $A$, and $\left\langle\mathcal{A}_{s}\right\rangle=Q_{k} \cap s^{-1} H s<A$, the group $H \cap\left(Q_{k} \cap s^{-1} H s\right)=H \cap s^{-1} H s$ has finite index in $Q_{k} \cap s^{-1} H s$. By Lemma 3.3 (with $Y=H$ and $g=s^{-1}$ ), the group $Q_{k} \cap s^{-1} H s$ has finite index in $s^{-1} H s$ and so $H \cap s^{-1} H s$ has finite index in $s^{-1} H s$ for all $s \in \mathcal{S}^{ \pm 1}$. Conjugating we have $s H s^{-1} \cap H$ has finite index in $H$ for all $s \in \mathcal{S}^{ \pm 1}$. Combining we have $s^{-1} H s \cap H$ has finite index in both $H$ and $s^{-1} H s$ for all $s \in \mathcal{S}^{ \pm 1}$.

Proof of Lemma 3.5 Now suppose $H$ has infinite index in $A$. The subcommensurated sequence $H=Q_{0} \prec Q_{1} \prec \cdots \prec Q_{k} \prec G$ has length $k+1$. Theorem 1.8 shows that if $k=0$, then $G$ is one-ended and semistable at $\infty$. Inductively, we assume that if $G^{\prime}$ is finitely generated and there is a subcommensurated sequence $H^{\prime}=Q_{0}^{\prime} \prec Q_{1}^{\prime} \prec \cdots \prec$ $Q_{k-1}^{\prime} \prec G^{\prime}$ of length $k$ such that $H^{\prime}$ is finitely generated and has infinite index in $G^{\prime}$, then $G^{\prime}$ is one-ended and semistable at $\infty$.

In our case, $H$ has infinite index in $A$, and the length $k$ subcommensurated series $H=Q_{0} \prec Q_{1} \cap A \prec \cdots \prec Q_{k-1} \cap A \prec A$ implies that $A$ is one-ended and semistable at $\infty$. Hence we may choose a finite set $P$ of $\mathcal{A}$-relations so that $\Gamma_{(A, \mathcal{A})}(P)$ is one-ended and semistable at $\infty$.

If $s \in \mathcal{S}^{ \pm 1}$ and $a \in \mathcal{A}_{s}$, then there is a $G$-relation of the form $a=s^{-1} a_{s} s$ for some $\mathcal{H}$-word $a_{s}$. Let $R$ be the (finite) collection of all such relations. Define

$$
\widetilde{\Gamma}:=\Gamma_{(G, \mathcal{A} \cup \mathcal{S})}(P \cup R) .
$$

We simultaneous show $\widetilde{\Gamma}$ is one-ended and semistable at $\infty$ by showing that all proper edge path rays in $\widetilde{\Gamma}$ are properly homotopic (completing the proof of the semistability part of Theorem 1.9).

Claim 3 Let $K$ be the length of the longest $R$-relation. If $v \in G$ (so $v$ is a vertex of $\widetilde{\Gamma}), s \in \mathcal{S}^{ \pm 1}$ and $r$ is an $\mathcal{A}_{s}$-proper ray at $v$, then $r$ is properly homotopic $\operatorname{rel}\{v\}$ to a ray of the form $\left(s^{-1}, h_{1}^{\prime}, h_{2}^{\prime}, \ldots\right)$, where $h_{i}^{\prime} \in \mathcal{H}$. Furthermore, this proper homotopy has image in $\mathrm{St}^{K}(\mathrm{im}(r))$.

Proof Suppose $r=\left(a_{1}, a_{2}, \ldots\right)$ with $a_{i} \in \mathcal{A}_{s}$. Then $r$ is properly homotopic $\operatorname{rel}\{v\}$ to $\left(s^{-1},\left(a_{1}\right)_{s}, s, s,{ }^{-1}\left(a_{2}\right)_{s}^{-1}, s, \ldots\right)$, simply by using the 2 -cells for the $R$-relation $a_{i}=s^{-1}\left(a_{i}\right)_{s} s$. Then $r$ is properly homotopic $\operatorname{rel}\{v\}$ to $\left(s^{-1},\left(a_{1}\right)_{s},\left(a_{2}\right)_{s}, \ldots\right)$ by a proper homotopy in $\mathrm{St}^{K}(\mathrm{im}(r))$. 
If $v \in G$ and $C_{v}$ is a compact subcomplex of $v \Gamma_{(A, \mathcal{A})}(P) \subset \widetilde{\Gamma}$ then there is a compact subcomplex $D_{v}$ of $v \Gamma_{(A, \mathcal{A})}(P)$ such that if $r$ and $s$ are edge path rays at $w \in v \Gamma_{(A, \mathcal{A})}(P)-D_{v}$, then $r$ and $s$ are properly homotopic rel\{w\} by a proper homotopy in $v \Gamma_{(A, \mathcal{A})}-C_{v}$. Hence, if $C$ is a compact subcomplex of $\widetilde{\Gamma}$ and we let $C_{v}:=C \cap v \Gamma_{(A, \mathcal{A})}(P)$ (for the finite set of vertices $v$ such $C \cap v \Gamma_{(A, \mathcal{A})}(P) \neq \varnothing$ ) and let $D:=\bigcup D_{v}$, then any two $\mathcal{A}$-rays $r$ and $s$ at $w \in v \Gamma_{(A, \mathcal{A})}(P)-D$ are properly homotopic $\operatorname{rel}\{w\}$ in $\widetilde{\Gamma}-C$.

[18, Lemma 2] is an elementary graph theory result that states: for each $v \in G$, there is an $\mathcal{H}$-ray $r_{v}$ at $v$ such that for any compact set $C \subset \widetilde{\Gamma}$ there are only finitely many $v \in G$ such that $r_{v}$ intersects $C$. Also, for each $s \in \mathcal{S}^{ \pm 1}$, there is an $\mathcal{A}_{s}$-ray $r_{(s, v)}$ at $v$ such that for any compact set $C \subset \widetilde{\Gamma}$, only finitely many $v$ are such that $r_{(s, v)}$ intersects $C$.

Choose a sequence of compact subcomplexes $\left\{C_{i}\right\}_{i=1}^{\infty}$ of $\widetilde{\Gamma}$ satisfying the following conditions:

(1) $\bigcup_{i=1}^{\infty} C_{i}=\widetilde{\Gamma}$.

(2) $\mathrm{St}^{K}\left(C_{i}\right)$ (see Claim 3) is contained in the interior of $C_{i+1}$, and the finite set of vertices $v$ such that $r_{v}$ or $r_{(s, v)}\left(s \in \mathcal{S}^{ \pm 1}\right)$ intersects $C_{i}$, is a subset of $C_{i+1}$.

(3) If $r$ and $s$ are $\mathcal{A}$-rays in $\widetilde{\Gamma}-C_{i}$ both based at a vertex $v$, then $r$ and $s$ are properly homotopic $\operatorname{rel}\{v\}$ by a proper homotopy in $v \Gamma_{(A, \mathcal{A})}(P)-C_{i-1}$; see Theorem 2.2(4).

For convenience, define $C_{i}:=\varnothing$ for $i<1$ and observe that conditions (1)-(3) (see Theorem 2.2(5)) remain valid for all $C_{i}$. The next lemma implies Lemma 3.5 and concludes the proof of the semistability part of Theorem 1.9.

Lemma 3.6 If $v$ is a vertex of $\widetilde{\Gamma}$, and $t=\left(e_{1}, e_{2}, \ldots\right)$ is an arbitrary ray at $v$, then $t$ is properly homotopic to $r_{v}, \operatorname{rel}\{v\}$.

Proof Assume that $t$ has consecutive vertices $v=v_{0}, v_{1}, \ldots$ By construction, if $v_{j} \in C_{i+1}-C_{i}$, then $r_{v_{j}}$ avoids $C_{i-1}$. Assume $j$ is the largest integer such that $C_{j}$ avoids $e_{i}$. Then $r_{v_{i-1}}$ and $r_{v_{i}}$ avoid $C_{j-1}$. We will show $r_{v_{i-1}}$ is properly homotopic to $e_{i} * r_{v_{i}}$ rel $\left\{v_{i-1}\right\}$ by a proper homotopy $H_{i}$ with image avoiding $C_{j-3}$.

If $e_{i} \in \mathcal{A}^{ \pm 1}$, this is clear by condition (3) with $H_{i}$ avoiding $C_{j-2}$. If $e_{i} \in \mathcal{S}^{ \pm 1}$, then $r_{v_{i-1}}$ and $r_{\left(e_{i}, v_{i-1}\right)}$ are $\mathcal{A}$-rays avoiding $C_{j-1}$, and so by (3) are properly homotopic $\operatorname{rel}\left\{v_{i-1}\right\}$ by a homotopy avoiding $C_{j-2}$. By Claim 3 and condition (2), $r_{\left(e_{i}, v_{i-1}\right)}$ is properly homotopic $\operatorname{rel}\left\{v_{i-1}\right\}$ to a ray $\left(e_{i}, h_{1}^{\prime}, h_{2}^{\prime}, \ldots\right)$, where $h_{i}^{\prime} \in \mathcal{H}^{ \pm 1}$ and the 


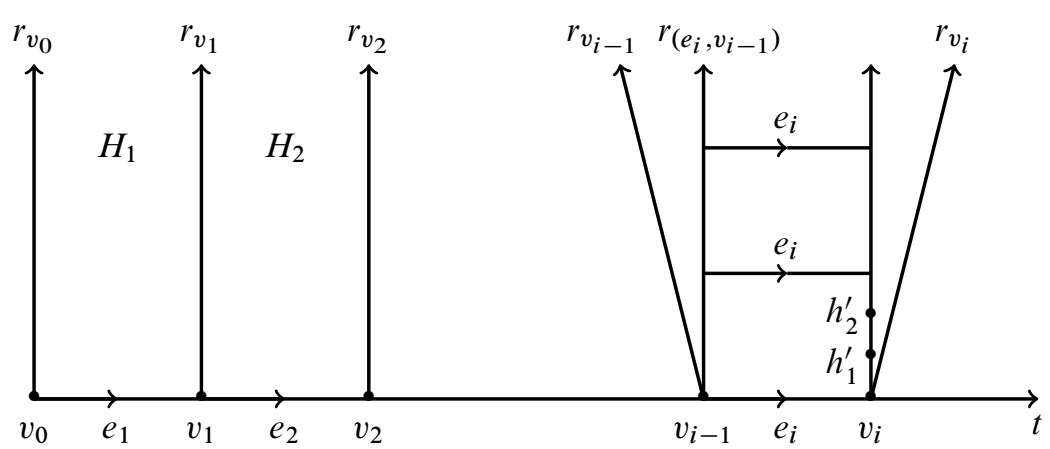

Figure 1: Inductive construction of $H$

homotopy avoids $C_{j-2}$. By condition (3), $\left(h_{1}^{\prime}, h_{2}^{\prime}, \ldots\right)$ is properly homotopic $\operatorname{rel}\left\{v_{i}\right\}$ to $r_{v_{i}}$ by a proper homotopy that avoids $C_{j-3}$. Patch these three proper homotopies together to obtain $H_{i}$; see Figure 1 .

Let $H$ be the homotopy $\operatorname{rel}\{v\}$ of $t$ to $r_{v}$, obtained by patching together the homotopies $H_{i}$. We need to check that $H$ is proper. Let $C \subset \widetilde{\Gamma}$ be compact. Choose an index $j$ such that $C \subseteq C_{j}$. Since $t$ is a proper edge path to $\infty$, choose an index $N$ such that all edges after the $N^{\text {th }}$ edge of $t$ avoid $C_{j+3}$. Then for all $i>N, H_{i}$ avoids $C_{j}$, so $H$ is proper.

This concludes the proof of Lemma 3.5 and the first part of Theorem 1.9.

\section{Simple connectivity at $\infty$}

It is straightforward to check that the proof of the simply connected at $\infty$ part of Theorem 1.8 given in [3] extends to the finitely generated case (as follows): if $\langle\mathcal{S}: R\rangle$ is a finite presentation of the group $G$ then $\Gamma_{(G, \mathcal{S})}(R)$ is simply connected. The only time the simple connectivity of $\Gamma$ is used in the proof of Theorem 1.8 is via this fact:

(*) If $C$ is a compact subset of $\Gamma$ and $N$ is a fixed positive integer, then there is an integer $M(N, C)$ such that any edge path loop $\alpha$ of length at most $N$ in $\Gamma-\mathrm{St}^{M}(C)$ is homotopically trivial in $\Gamma-C$.

Suppose $G$ is a finitely generated subgroup of a finitely presented group $W$ and $W$ has presentation $\langle\mathcal{W}: R\rangle$, where $\mathcal{W}$ contains a set of generators $\mathcal{G}$ for $G$. When proving a finitely generated version of the simply connected at $\infty$ part of Theorem 1.8, all work is done in the simply connected space $\Gamma_{(W, \mathcal{W})}(R)$, and one only needs $(*)$ for edge path loops $\alpha$ with edge labels in $\mathcal{G}^{ \pm 1}$. Hence, the proof of the simply connected at $\infty$ part of Theorem 1.8 directly extends to the stronger finitely generated version. 
Theorem 4.1 (Conner and Mihalik improved) Suppose $H$ is a one-ended, finitely presented infinite subgroup of infinite index in the finitely generated and recursively presented group $G$. If $H$ is commensurated in $G$, then $G$ is one-ended and simply connected at $\infty$.

In order to finish the proof of our main theorem it remains to prove the following.

Theorem 4.2 Suppose that $H$ is a one-ended finitely presented subcommensurated subgroup of infinite index in the finitely generated and recursively presented group $G$ :

$$
H=Q_{0} \prec Q_{1} \prec \cdots \prec Q_{k} \prec Q_{k+1}=G .
$$

Then $G$ is simply connected at $\infty$.

Proof We say $H$ is $(k+1)$-subcommensurated in $G$. When $k=0$, Theorem 4.1 implies that $G$ is simply connected at $\infty$. Assume (inductively) the statement of Theorem 4.2 is valid when $H$ is $(n+1)$-subcommensurated for $n<k$. Let

$$
\begin{aligned}
\mathcal{H} & :=\left\{h_{1}, \ldots, h_{n}\right\} \text { generate } H, \\
\mathcal{G} & :=\left\{h_{1}, \ldots, h_{n}, s_{1}, \ldots, s_{m}\right\} \text { generate } G, \text { and } \\
\mathcal{S} & :=\left\{s_{1}, \ldots, s_{m}\right\} .
\end{aligned}
$$

For $p$ an element of a group $P$ with generating set $\mathcal{P}$, let $|g|_{\mathcal{P}}$ be word length in $\mathcal{P}$ : the smallest integer $\ell$ such that $g=p_{1} \cdots p_{\ell}$ where $p_{i} \in \mathcal{P}^{ \pm 1}$. We use the notation $|g|:=|g|_{\mathcal{P}}$ for all $g \in P$.

For each $s \in \mathcal{S}^{ \pm}$let $\mathcal{A}_{s}$ be a finite generating set for $s H s^{-1} \cap Q_{k}$ (see Lemma 3.3 with $Y=H$ ) and let $\mathcal{A}_{s}^{\prime}:=s^{-1} \mathcal{A}_{s} \subset \subset H$. Choose an integer $L_{1}$ such that

We have that

$$
L_{1} \geq|a|_{\mathcal{H}} \quad \text { for all } a \in \bigcup_{s \in \mathcal{S}^{ \pm 1}} \mathcal{A}_{s}^{\prime} .
$$

$$
A_{s}:=\left\langle\mathcal{A}_{s}\right\rangle<Q_{k}
$$

has finite index in $s H s^{-1}$, and

$$
A_{s}^{\prime}:=\left\langle\mathcal{A}_{s}^{\prime}\right\rangle=s^{-1} A_{s} s<H
$$

has finite index in $H$. As in Section 3, define

$$
\mathcal{A}_{1}:=\bigcup_{s \in \mathcal{S}^{ \pm 1}} \mathcal{A}_{s}, \quad \mathcal{A}:=\mathcal{H} \cup \mathcal{A}_{1} \quad \text { and } \quad A:=\langle\mathcal{A}\rangle<Q_{k} .
$$

For each $s \in \mathcal{S}^{ \pm 1}$ and $a \in \mathcal{A}_{s}$ there is an $\mathcal{H}$-word $w(s, a)$ of length at most $L_{1}$, such that $s^{-1} a s w^{-1}(a, s)$ is an $(A, \mathcal{A})$-relator, which we denote by $r(a, s)$. Let

$$
R_{1}:=\left\{r(a, s) \mid s \in \mathcal{S}^{ \pm 1}, a \in \mathcal{A}_{s}\right\} .
$$


For each $g \in G$ let $\mathcal{B}_{g}$ be a finite generating set for the group $g A g^{-1} \cap Q_{k}$; see Lemma 3.3 with $Y=A$. Let $\mathcal{B}_{g}^{\prime}:=g^{-1} \mathcal{B}_{g} g \subset A \cap g^{-1} Q_{k} g$. If $g$ is in $A$, then $g A g^{-1}=A$ and so we define $\mathcal{B}_{g}:=\mathcal{A}:=\mathcal{B}_{g}^{\prime}$. Then

$$
B_{g}:=\left\langle\mathcal{B}_{g}\right\rangle=g A g^{-1} \cap Q_{k}
$$

has finite index in $\mathrm{gAg}^{-1}$, and

$$
B_{g}^{\prime}:=\left\langle\mathcal{B}_{g}^{\prime}\right\rangle=g^{-1} B_{g} g=A \cap g^{-1} Q_{k} g
$$

has finite index in $A$. For each $g \in G$, let $N_{g}$ be an integer so that in the Cayley graph $\Gamma_{(G, \mathcal{G})}$, each vertex of $A$ is within $N_{g}$ of a vertex of $B_{g}^{\prime}$. Let

$$
\mathcal{B}_{j}:=\mathcal{A} \cup\left(\bigcup_{\{g \in G:|g| \leq j\}} \mathcal{B}_{g}\right) \subset Q_{k} \quad \text { and } \quad N_{j}:=\max \left\{N_{g}: g \in G \text { and }|g| \leq j\right\} .
$$

Lemma 4.3 Suppose $g$ is in $G$ and $y$ in $g A$. Then in $\Gamma_{(G, \mathcal{G})}, y$ is within $N_{g}+|g|$ of a point of $B_{g}$.

Proof Let $y=g a$ for some $a \in A$. There is $b^{\prime} \in B_{g}^{\prime}=g^{-1} Q_{k} g \cap A$ within $N_{g}$ of $a$. Then $y^{\prime}:=g b^{\prime}$ is within $N_{g}$ of $y=g a$. As $y^{\prime} g^{-1}=g b^{\prime} g^{-1} \in Q_{k} \cap g A g^{-1}=B_{g}$, $y^{\prime}$ is within $|g|$ of $B_{g}$ and so $y$ is within $N_{g}+|g|$ of $B_{g}$.

If $H$ has finite index in $A$, then by Lemma 3.4, $H$ is commensurated in $G$ and so $G$ is simply connected at $\infty$ by Theorem 4.1. So we may assume that $H$ has infinite index in $A$. Our induction hypothesis, Lemma 3.2 and the results of Section 3 imply the following.

Lemma 4.4 The finitely generated subgroups $A$ and $B_{j}:=\left\langle\mathcal{B}_{j}\right\rangle$ of $Q_{k}$ are one-ended, semistable at $\infty$ and simply connected at $\infty$ for all $j \geq 1$.

Next assume that $G$ is a subgroup of a finitely presented (over) group $W$. Then for all $j \geq 1, A$ and $B_{j}$ are simply connected at $\infty$ in $W$. Let $\mathcal{W}$ be a finite generating set for $W$ containing $\mathcal{A}$ and $\mathcal{G}$, and let $\langle\mathcal{W}: R\rangle$ be a finite presentation for $W$. Assume that $R$ contains a set $R^{\prime}$ of $\mathcal{A}$-relations so that $\Gamma_{(A, \mathcal{A})}\left(R^{\prime}\right)$ is semistable at $\infty$. We also assume that $R^{\prime}$ contains the set of conjugation relations $R_{1}$. If $v$ is a $G$-vertex of $\Gamma_{(W, \mathcal{W})}(R)$, let $v \Gamma_{(A, \mathcal{A})}\left(R^{\prime}\right)$ be the copy of $\Gamma_{(A, \mathcal{A})}\left(R^{\prime}\right)$ at $v$. To ease notation, if $p$ is a $\mathcal{G}^{ \pm 1}$-word and $\bar{p}$ is the corresponding element of $G$, define $B_{p}:=B_{\bar{p}}$ and $B_{p}^{\prime}:=B_{\bar{p}}^{\prime}$.

As a direct consequence of Lemma 4.3, we have another lemma. 
Lemma 4.5 Suppose $v$ is a $G$-vertex of $\Gamma_{(W, \mathcal{W})}(R)$ and $\left(e_{1}, \ldots, e_{i}\right)$ labels a $\mathcal{G}$-edge path with consecutive vertices $v=v_{0}, v_{1}, \ldots, v_{i}$. If $j \in\{1,2, \ldots, i\}$ and $w$ is a vertex of the Cayley graph $v_{j} \Gamma_{(A, \mathcal{A})}$, then $w$ is within $j+N_{j}$ of a vertex of $v \Gamma_{\left(B_{j}, \mathcal{B}_{j}\right)}$.

Note that Theorem 4.2 does not follow directly from Lemma 2.9 and the fact that $B_{\ell}$ is simply connected at $\infty$ in $W$ for all $\ell$, but it does follow from the next lemma.

Lemma 4.6 Given any compact subcomplex $C$ of $\Gamma_{(W, \mathcal{W})}(R)$ there is a compact subcomplex $D$ of $\Gamma_{(W, \mathcal{W})}(R)$ such that any $\mathcal{G}$-loop $\alpha$ at a $G$-vertex of $\Gamma_{(W, \mathcal{W})}(R)-D$ is homotopically trivial in $\Gamma_{(W, \mathcal{W})}(R)-C$.

Proof If $v$ is a vertex of $\Gamma_{(W, \mathcal{W})}(R)$ and $v \Gamma_{(A, \mathcal{A})}\left(R^{\prime}\right) \cap C$ is empty, then by Theorem 2.2(5), any two $A$-rays at $v$ are properly homotopic relative to $v$ by a proper homotopy in $v \Gamma_{(A, \mathcal{A})}\left(R^{\prime}\right)$ (and so the homotopy avoids $C$ ). There are only finitely many $v \Gamma_{(A, \mathcal{A})}\left(R^{\prime}\right)$ that intersect $C$. Since $\Gamma_{(A, \mathcal{A})}\left(R^{\prime}\right)$ is semistable at $\infty$ there is a compact subcomplex $D_{1}$ of $\Gamma_{(W, \mathcal{W})}(R)$ such that any two $\mathcal{A}$-edge path rays based at a $G$-vertex $v$ and with image in $\Gamma_{(W, \mathcal{W})}(R)-D_{1}$, are properly homotopic relative to $v$ by a homotopy in $v \Gamma_{(A, \mathcal{A})}\left(R^{\prime}\right)-C$. There are only finitely many Cayley graphs of the form $v \Gamma_{(H, \mathcal{H})}$ or $v \Gamma_{\left(A_{s}, \mathcal{A}_{s}\right)}$ (for $v \in G$ and $s \in \mathcal{S}^{ \pm 1}$ ) that intersect $D_{1}$. Choose a finite subcomplex $D$ of $\Gamma_{(W, \mathcal{W})}(R)$ such that $D$ contains $D_{1}$ and the bounded components of both $v \Gamma_{\left(A_{s}, \mathcal{A}_{s}\right)}-\mathrm{St}^{L_{1}}\left(D_{1}\right)$ and $v \Gamma_{(H, \mathcal{H})}-D_{1}$, for all $v \Gamma_{\left(A_{s}, \mathcal{A}_{s}\right)}$ and $v \Gamma_{(H, \mathcal{H})}$ that intersect $D_{1}$. (Recall, if $s \in \mathcal{S}^{ \pm 1}$ and $a \in \mathcal{A}_{s}$, then there is an $\mathcal{H}$-word $w(a, s)$ of length at most $L_{1}$, such that $s^{-1} a s w(a, s)^{-1} \in R_{1}$.)

Therefore,

(*) if $e$ is an edge of $\Gamma_{(W, \mathcal{W})}(R)-D$ with initial vertex $v \in G$, terminal vertex $w$ and label $s \in \mathcal{S}^{ \pm 1}$, then there is a proper $A_{s}$-ray $q_{v}$ at $v$ avoiding $\operatorname{St}^{L_{1}}\left(D_{1}\right)$ and hence an $\mathcal{H}$-ray $s_{w}$ at $w$ avoiding $D_{1}$, such that $q_{v}$ and $\left(e, s_{w}\right)$ are homotopic relative to $v$ by a homotopy (using only 2 -cells arising from $R_{1}$-conjugation relations) in $\Gamma_{(W, \mathcal{W})}(R)-C$ (see Figure 2); and

$(* *)$ if $v$ is a $G$-vertex of $\Gamma_{(W, \mathcal{W})}(R)-D$, there is an $\mathcal{H}$-ray at $v$ in $\Gamma_{(W, \mathcal{W})}(R)-D_{1}$.

Assume $\alpha$ is a $\mathcal{G}$-loop based at the $G$-vertex $v$ in $\Gamma_{(W, \mathcal{W})}(R)-D$. We wish to show that $\alpha$ is homotopically trivial in $\Gamma_{(W, \mathcal{W})}-C$. Since $G$ is one-ended, we may assume that $\Gamma_{(G, \mathcal{G})}-D$ is connected, and so there is an edge path in $\Gamma_{(G, \mathcal{G})}-D$ from $v$ to a vertex of $H$. Hence we assume, without loss, that $v \in H$. Let $\ell$ be the length of $\alpha$. By Lemma $4.4, B_{\ell}$ is simply connected at $\infty$ and Lemma 2.9 implies that there is a compact subcomplex $E\left(C, \ell+N_{\ell}\right)$ of $\Gamma_{(W, \mathcal{W})}(R)$ with the following property: if $\beta$ is an edge path loop with image in $\Gamma_{(W, \mathcal{W})}(R)-E$ and each vertex of $\beta$ is within $\ell+N_{\ell}$ of $B_{\ell}$, then $\beta$ is homotopically trivial in $\Gamma_{(W, \mathcal{W})}(R)-C$. 


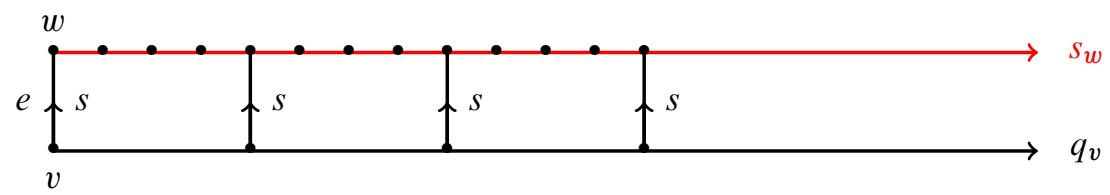

Figure 2: For $e$ an edge of $\Gamma_{(W, \mathcal{W})}(R)-D$, there is a proper $A_{s}$-ray $q_{v}$ avoiding $\mathrm{St}^{L_{1}}\left(D_{1}\right)$ and hence an $\mathcal{H}$-ray $s_{w}$ avoiding $D_{1}$, such that $q_{v}$ and $\left(e, s_{w}\right)$ are homotopic in $\Gamma_{(W, \mathcal{W})}(R)-C$.

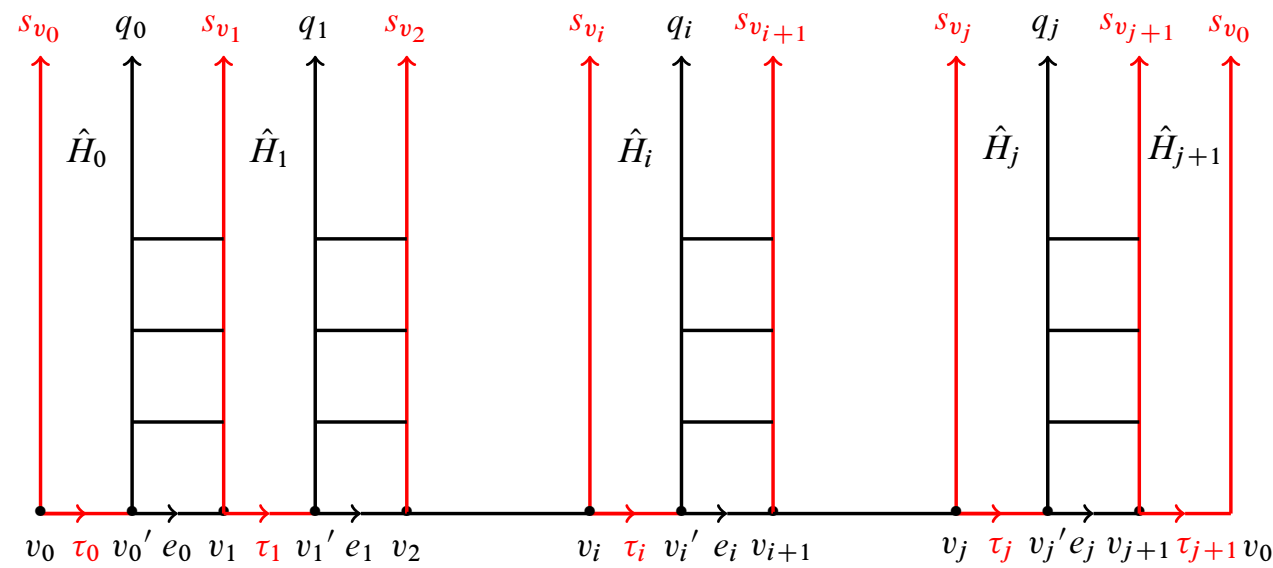

Figure 3: A $\mathcal{G}$-loop based at a $G$-vertex in $\Gamma_{(W, \mathcal{W})}(R)-D$ is homotopically trivial in $\Gamma_{(W, \mathcal{W})}(R)-C$.

It is enough to show that $\alpha$ is homotopic to such a $\beta$ in $\Gamma_{(W, \mathcal{W})}(R)-C$ (and this is where the semistability of $A$ comes in). Let $s_{v}$ be an $\mathcal{H}$-proper edge path ray at $v$ in $\Gamma_{(W, \mathcal{W})}(R)-D_{1}$; see $(* *)$. If all edges of $\alpha$ are $\mathcal{H}$-edges, then by the definition of $D_{1}$, the rays $s_{v_{0}}$ and $\left(\alpha, s_{v_{0}}\right)$ are properly homotopic relative to $v$, by $\hat{H}$ a proper homotopy with image in $v \Gamma_{(A, \mathcal{A})}\left(R^{\prime}\right)-C$. Otherwise, write $\alpha$ as $\left(\tau_{0}, e_{0}, \tau_{1}, e_{1}, \ldots, \tau_{j}, e_{j}, \tau_{j+1}\right)$, where $\tau_{i}$ is a (possibly trivial) $\mathcal{H}$-path and $e_{i}$ is an $\mathcal{S}$-edge. Let the initial vertex of $e_{i}$ be $v_{i}^{\prime}$ and the terminal vertex of $e_{i}$ be $v_{i+1}$; see Figure 3.

If $e_{i}$ is labeled by $s \in \mathcal{S}^{ \pm 1}$ then let $q_{i}$ be a proper $\mathcal{A}_{s}$-ray at $v_{i}^{\prime}$, avoiding $\operatorname{St}^{L_{1}}\left(D_{1}\right)$. For each edge $a$ of $q_{i}$, there is a 2-cell with boundary label $\left(s^{-1}, a, s, w^{-1}(a, s)\right)$, where $w(a, s)$ is an $\mathcal{H}$-word of length at most $L_{1}$ (see the definition of $R_{1}$ ). So if $q_{i}$ is labeled $\left(a_{1}, a_{2}, \ldots\right)$ then $s_{v_{i+1}}$, the $\mathcal{H}$-ray at $v_{i+1}$, with labeling $\left(w\left(a_{1}, s\right), w\left(a_{2}, s\right), \ldots\right)$ is such that $q_{i}$ is properly homotopic to $\left(e_{i}, s_{v_{i+1}}\right)$ relative to $v_{i}^{\prime}$ by a homotopy (only using $R_{1}$-cells) with image in $\Gamma_{(W, \mathcal{W})}(R)-C$. The ray $s_{v_{i}}$ has image in $\Gamma_{(W, \mathcal{W})}(R)-D_{1}$ and so by the semistability of $\Gamma_{(A, \mathcal{A})}\left(R^{\prime}\right)$ we have for $i \in\{0,1, \ldots, j\}$, 
$q_{i}$ is properly homotopic to $\left(\tau_{i}^{-1}, s_{v_{i}}\right)$ relative to $v_{i}^{\prime}$ by a proper homotopy $\hat{H}_{i}$ in $v_{i}^{\prime} \Gamma_{(A, \mathcal{A})}-C$. Finally, define $\hat{H}_{j+1}$ to be a proper homotopy in $v_{0} \Gamma_{(A, \mathcal{A})}-C$ of $s_{v_{0}}$ to $\left(\tau_{j+1}^{-1}, s_{v_{j+1}}\right)$.

Assume that $v_{i}^{\prime}$ is the $j(i)^{\text {th }}$ vertex of $\alpha$. By Lemma 4.5, every vertex of $v_{i}^{\prime} \Gamma_{(A, \mathcal{A})}\left(R^{\prime}\right)$ is within $N_{j(i)}+j(i)\left(\leq N_{\ell}+\ell\right)$ of $B_{j(i)} \subset B_{\ell}$. Hence for $i \in\{0, \ldots, j\}$, each vertex of (the image of) $\hat{H}_{i}$ is within $N_{\ell}+\ell$ of a vertex of $B_{\ell}$.

Combining the homotopies $\hat{H}_{0}, \ldots, \hat{H}_{j+1}$ along with those of $q_{i}$ to $\left(e_{i}, s_{v_{i+1}}\right)$, we have a proper cellular homotopy $\hat{H}$ (relative to $v)$ of $s_{v_{0}}$ to $\left(\alpha, s_{v_{0}}\right)$ with image in $\Gamma_{(W, \mathcal{W})}(R)-C$, and each vertex of the image of the homotopy $\hat{H}$ is within $N_{\ell}+\ell$ of a vertex of $B_{\ell}$. Then

$$
\hat{H}:[0,1] \times[0, \infty) \rightarrow \Gamma_{(W, \mathcal{W})}(R)-C
$$

such that $\left.\hat{H}\right|_{[0,1] \times\{0\}}$ is $\alpha,\left.\hat{H}\right|_{\{0\} \times[0, \infty)}$ and $\left.\hat{H}\right|_{\{1\} \times[0, \infty)}$ both agree with $s_{v_{0}}$, and each vertex of the image of $\hat{H}$ is within $N_{\ell}+\ell$ of $B_{\ell}$. Choose $N$ such that $\hat{H}^{-1}(E) \subset$ $[0,1] \times[0, N]$. The loop $\left.\hat{H}\right|_{[0,1] \times\{N+1\}}$ provides a $\mathcal{G}$-loop $\beta$ such that $\alpha$ is homotopic to $\beta$ in $\Gamma_{(W, \mathcal{W})}(R)-C$, and each vertex of $\beta$ is within $N_{\ell}+\ell$ of $B_{\ell}$. By the definition of $E$, the loop $\beta$ (and hence $\alpha$ ) is homotopically trivial in $\Gamma_{(W, \mathcal{W})}(R)-C$.

This completes the proof of Theorem 4.2.

\section{Grigorchuk's group is simply connected at $\infty$}

In 1985, Mihalik [17] proved that if $H$ is an ascending HNN-extension of a finitely presented group $G$, then $H$ is semistable at $\infty$. If, additionally, $G$ is one-ended, then $H$ is simply connected at $\infty$. Finitely presented ascending HNN extensions of one-ended finitely generated groups are not even known to be semistable at $\infty$.

Grigorchuk [7;8] constructed an infinite finitely generated torsion group $G$. The group $G$ has a subgroup of finite index $T$, and $T$ has a subgroup of finite index that is isomorphic to $T \times T$. There is a finitely presented ascending HNN-extension of $G$ (and so $G$ is recursively presented). It is easy to see that if $G$ is finitely generated and $H$ has finite index in $G$, then $G$ is semistable at $\infty$ if and only if $H$ is semistable at $\infty$; see [5, Proposition 16.5.3]. [16, Theorem 2.2] implies that $T \times T$ (and hence $G$ ) is semistable at $\infty$. Theorem 5.1 implies that Grigorchuk's group $G$ is simply connected at $\infty$ and Theorem 5.2 implies that the finitely presented HNN-extension of $G$ is also simply connected at $\infty$. This last result generalizes the main result of [4] which states that $G$ is quasisimply filtered (qsf). 
Theorem 5.1 Suppose the recursively presented group $G$ is finitely generated and isomorphic to $A \times B$ where $A$ and $B$ are finitely generated infinite groups and $A$ is one-ended. Then $G$ is simply connected at $\infty$.

Proof Suppose $G$ is a subgroup of a finitely presented group $Q$. Let $\mathcal{P}:=\left\langle\mathcal{Q}: R_{Q}\right\rangle$ be a finite presentation for $Q$ such that $\mathcal{Q}$ contains generators $\mathcal{A}$ for $A$ and $\mathcal{B}$ for $B$, and $R_{Q}$ contains the commutation relations $R$ between these generators. Define $\Gamma(\mathcal{P}):=\Gamma_{(Q, \mathcal{Q})}\left(R_{Q}\right)$ (the Cayley 2-complex for $\left.\mathcal{P}\right)$. The subspace $\Gamma_{(G, \mathcal{A} \cup \mathcal{B})}(R) \subset$ $\Gamma(\mathcal{P})$ is $G$-equivariantly homeomorphic to $\Gamma_{(A, \mathcal{A})} \times \Gamma_{(B, \mathcal{B})}$. Let $\phi_{A}: \Gamma_{(G, \mathcal{A} \cup \mathcal{B})}(R) \rightarrow$ $\Gamma_{(A, \mathcal{A})}$ and $\phi_{B}: \Gamma_{(G, \mathcal{A} \cup \mathcal{B})}(R) \rightarrow \Gamma_{(B, \mathcal{B})}$ be projections.

Claim 5.1.1 Suppose $C$ is a compact subcomplex of $\Gamma(\mathcal{P})$. Then there is a compact subcomplex $D$ of $\Gamma(\mathcal{P})$ such that if $\gamma$ is an edge path loop in $\Gamma_{(G, \mathcal{A} \cup \mathcal{B})}(R)-D$ with edge labels all in $\mathcal{A}^{ \pm 1}$ or all in $\mathcal{B}^{ \pm 1}$, then $\gamma$ is homotopically trivial in $\Gamma(\mathcal{P})-C$.

Proof The set $\phi_{B}(C)$ union the bounded components of $\Gamma_{(B, \mathcal{B})}-\phi_{B}(C)$ is compact and so contains finitely many vertices. If $v$ is one of these vertices, let $\beta_{v}$ be an edge path from $v$ to a vertex $v^{\prime}$ of an unbounded path component of $\Gamma_{(B, \mathcal{B})}-\phi_{B}(C)$. If $v$ is a vertex of an unbounded component of $\Gamma_{(B, \mathcal{B})}-\phi_{B}(C)$, then $\beta_{v}$ is trivial and $v^{\prime}:=v$. In any case, let $\tau_{v}$ be a proper edge path ray at $v^{\prime}$ with image in $\Gamma_{(B, \mathcal{B})}-\phi_{B}(C)$. Let $L$ be the length of the longest $\beta_{v}$. Let $D:=\mathrm{St}^{L}(C)$. Suppose each edge of $\gamma$ has label in $\mathcal{A}^{ \pm 1}$. Then $\phi_{B}(\gamma)$ is a single vertex of $v$ of $\Gamma_{(B, \mathcal{B})}$. Let $w$ be the initial vertex of $\gamma$, and lift the ray $\left(\beta_{v}, \tau_{v}\right)$ to $w$ (ie consider the $\mathcal{B}$-ray at $w$ with the same edge labeling as that of $\left.\left(\beta_{v}, \tau_{v}\right)\right)$. Call the lift $\left(\tilde{\beta}_{v}, \tilde{\tau}_{v}\right)$. Since each edge of $\left(\tilde{\beta}_{v}, \tilde{\tau}_{v}\right)$ has label in $\mathcal{B}^{ \pm 1}$ and each edge of $\gamma$ has label in $\mathcal{A}^{ \pm 1}$, there is a proper homotopy of $H:[0,1] \times[0, \infty) \rightarrow \Gamma_{(G, \mathcal{A} \cup \mathcal{B})}(R)$ that uses the product structure of $\Gamma_{(G, \mathcal{A} \cup \mathcal{B})}(R)$ to basically slide the loop $\gamma$ along the ray $\left(\tilde{\beta}_{v}, \tilde{\tau}_{v}\right)$. In particular, $\left.H\right|_{\{0\} \times[0, \infty)}=\left.H\right|_{\{1\} \times[0, \infty)}=\left(\tilde{\beta}_{v}, \tilde{\tau}_{v}\right)$ and $\left.H\right|_{[0,1] \times\{n\}}$ is an edge path loop with the same labeling as $\gamma$ for each integer $n \geq 0$. The image of $\phi_{B}(H)$ is simply the image of $\left(\beta_{v}, \tau_{v}\right)$. Say $\beta_{v}$ has length $K(\leq L)$. Then $\left.H\right|_{[0,1] \times[0, K]}$ has image in $\operatorname{St}^{K}(\operatorname{image}(\gamma)) \subset \Gamma_{(G, \mathcal{A} \cup \mathcal{B})}(R)-C$. As $\phi_{B}\left(\left.H\right|_{[0,1] \times[K, \infty)}\right)$ has the same image as $\tau_{v}$ (which avoids $\phi_{B}(C)$ ), the image of $H$ avoids $C$. Note that $\left.H\right|_{[0,1] \times\{n\}}$ is the translate of $\gamma$ to the $n^{\text {th }}$ vertex of $\left(\tilde{\beta}_{v}, \tilde{\tau}_{v}\right)$. Since this ray is proper, there is an integer $N$ such that the translate of $\gamma$ to the $N^{\text {th }}$ vertex of $\left(\tilde{\beta}_{v}, \tilde{\tau}_{v}\right)$ is homotopically trivial in $\Gamma(\mathcal{P})-C$. Since $\left.H\right|_{[0,1] \times[0, N]}$ provides a homotopy (avoiding $C$ ) of $\gamma$ to a loop that bounds in $\Gamma(\mathcal{P})-C$, we have that $\gamma$ is homotopically trivial in $\Gamma(\mathcal{P})-C$.

A completely analogous argument in the case all edges of $\gamma$ have labels in $\mathcal{B}^{ \pm 1}$ finishes the proof of the claim. Note that the one-endedness of $A$ is not important yet. 
Let $C$ be a compact subcomplex of $\Gamma(\mathcal{P})$ and $D$ the associated compact set of Claim 5.1.1. Since $A$ is one-ended, $\Gamma_{(A, \mathcal{A})}-\phi_{A}(D)$ has one unbounded component $K$ and $\Gamma_{(A, \mathcal{A})}-K$ is compact. Let $z_{0}$ be a vertex of $K$. For each vertex $v$ of $\Gamma_{(A, \mathcal{A})}$ choose an edge path $\tau_{v}$ from $v$ to $z_{0}$ so that if $v \in K$ then $\tau_{v}$ has image in $K$. There are only finitely many vertices of $\Gamma_{(A, \mathcal{A})}$ not in $K$. Of these, let $M$ be the length of the longest $\tau_{v}$. Let $E:=\mathrm{St}^{M}(D)$. We show that edge path loops in $\Gamma_{(G, \mathcal{A} \cup \mathcal{B})}(R)-E$ are homotopically trivial in $\Gamma(\mathcal{P})-C$.

Let $\gamma$ be a loop in $\Gamma_{(G, \mathcal{A} \cup \mathcal{B})}(R)-E$. Write $\gamma$ as $\left(\alpha_{1}, \delta_{1}, \ldots, \alpha_{n}, \delta_{n}\right)$, where edge labels in the edge paths $\alpha_{i}$ (respectively $\delta_{i}$ ) are in $\mathcal{A}^{ \pm 1}$ (respectively $\mathcal{B}^{ \pm 1}$ ). Let $a_{i}$ be the initial vertex of $\alpha_{i}$ and $d_{i}$ the initial vertex of $\delta_{i}$. Let $a_{i}^{\prime}:=\phi_{A}\left(a_{i}\right)$ and $d_{i}^{\prime}:=\phi_{A}\left(d_{i}\right)$. Then $d_{i}^{\prime}=a_{i+1}^{\prime}$ since $\delta_{i}$ is a $\mathcal{B}$-path. Let $\tilde{\tau}_{i}$ be the lift of the path $\tau_{a_{i}^{\prime}}$ to $a_{i}$ and $\hat{\tau}_{i}$ be the lift of $\tau_{d_{i}^{\prime}}$ to $d_{i}$; see Figure 4. Note that

(1) $\tau_{d_{i}^{\prime}}=\tau_{a_{i+1}^{\prime}}$, so $\hat{\tau}_{i}$ and $\tilde{\tau}_{i+1}$ have the same edge labeling,

(2) $\tilde{\tau}_{i}$ and $\hat{\tau}_{i}$ share the same end point $v_{i}$, where $\phi_{A}\left(v_{i}\right)=z_{0} \in K$, and

(3) the translate of $\delta_{i}$ to $v_{i}$ (call it $\delta_{i}^{\prime}$ ) ends at $v_{i+1}$.

By (1) there is a product homotopy $H_{i}$ of $\left(\delta_{i}, \tilde{\tau}_{i+1}\right)$ to $\left(\hat{\tau}_{i}, \delta_{i}^{\prime}\right)$. We show that the image of $H_{i}$ avoids $D$.

If $d_{i}^{\prime}\left(=\phi_{A}\left(d_{i}\right)\right) \in K$ then the image of $\phi_{A}\left(H_{i}\right)$ is equal to the image of $\tau_{d_{i}^{\prime}}$ which is a subset of $K \subset \Gamma_{(A, \mathcal{A})}-\phi_{A}(D)$. In this case, $H_{i}$ has image in $\Gamma_{(G, \mathcal{A} \cup \mathcal{B})}(R)-D$. If $\phi_{A}\left(d_{i}\right) \notin K$, then the length of $\hat{\tau}_{i}$ is $\leq M$ and the image of $H_{i}$ is in $\operatorname{St}^{M}$ (image $\left.\left(\delta_{i}\right)\right) \subset$ $\Gamma_{(G, \mathcal{A} \cup \mathcal{B})}(R)-D\left(\right.$ since $\delta_{i}$ has image in $\Gamma_{(G, \mathcal{A} \cup \mathcal{B})}(R)-E$ and $\left.E=\mathrm{St}^{M}(D)\right)$.

The $\mathcal{A}$-loops $\left(\alpha_{i}, \hat{\tau}_{i}, \tilde{\tau}_{i}^{-1}\right)$ have image in $\Gamma_{(G, \mathcal{A} \cup \mathcal{B})}(R)-D$ and so, by Claim 5.1.1 are homotopically trivial by a homotopy $H_{i}^{\prime}$ in $\Gamma(\mathcal{P})-C$. Combining all homotopies $H_{i}$ and $H_{i}^{\prime}$ we have that $\gamma$ is homotopic to $\left(\delta_{1}^{\prime}, \ldots, \delta_{n}^{\prime}\right)$ by a homotopy in $\Gamma_{(G, \mathcal{A} \cup \mathcal{B})}(R)-D$. Since all edge labels of this last loop are in $\mathcal{B}^{ \pm 1}$, Claim 5.1.1 implies $\left(\delta_{1}^{\prime}, \ldots, \delta_{n}^{\prime}\right)$ (and hence $\gamma$ ) is homotopically trivial in $\Gamma(\mathcal{P})-C$.

If $\phi: G \rightarrow G$ is a monomorphism then the HNN extension

$$
H:=\left\langle G, t: t^{-1} g t=\phi(g) \text { for all } g \in G\right\rangle
$$

is called an ascending HNN extension of $G$ with stable letter $t$, and denoted $G *_{\phi, t}$. When $\phi$ is not an epimorphism, then $H$ is a strictly ascending HNN extension. There is an ascending HNN extension $H$ of Grigorchuk's group $G$ that is finitely presented. The main result of [4] shows that $H$ has the quasisimply filtered (qsf) property. Simple connectivity at $\infty$ implies qsf [1]. Our next result implies that $H$ is simply connected at $\infty$ and so generalizes [4]. We need not assume that $H$ is finitely presented. 


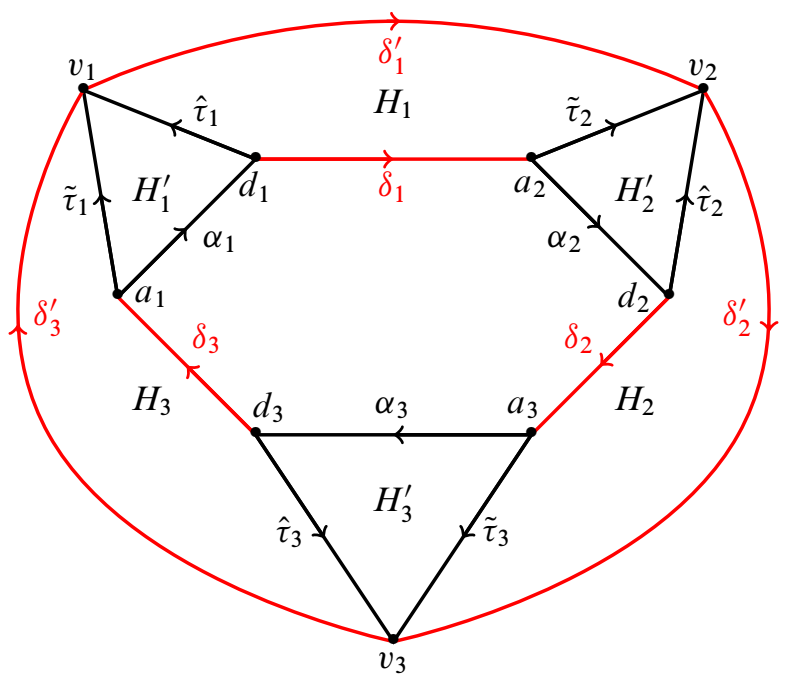

Figure 4: Loops in $\Gamma_{(G, \mathcal{A} \cup \mathcal{B})}(R)-E$ are homotopically trivial in $\Gamma(\mathcal{P})-C$.

Theorem 5.2 Suppose $H$ is an ascending $H N N$ extension of a one-ended finitely generated semistable at $\infty$ and simply connected at $\infty$ group $G$. Then $H$ is simply connected at $\infty$.

Proof Say $H:=G *_{\phi, t}$. Assume that $Q$ is finitely presented and $H$ is a subgroup of $Q$. We must show that $H$ is simply connected at $\infty$ in $Q$. Let $\mathcal{G}$ be a finite set of generators for $G$, and $R_{1}$ a finite set of relations so that $\Gamma_{(G, \mathcal{G})}\left(R_{1}\right)$ is semistable at $\infty$. Since $t$ is the stable letter in $G *_{\phi, t}$, the finite set $\mathcal{H}:=\mathcal{G} \cup\{t\}$ generates $H$. For each generator $g \in \mathcal{G}$ there is a $\mathcal{G}$-word (usually denoted $\phi(g)$ ) giving rise to the relator $t^{-1} g t=\phi(g)$. These (finitely many) relators are called the conjugation relations of $H$ with respect to $\mathcal{G}$. Let $R_{2}$ be this set of conjugation relations and let $R:=R_{1} \cup R_{2}$. Let $\mathcal{P}$ be a finite presentation for $Q$ with $\mathcal{H}$ a subset of the generators and $R$ a subset of the relations of $\mathcal{P}$. We assume $\Gamma_{(G, \mathcal{G})}\left(R_{1}\right)$ is a subset of $\Gamma_{(H, \mathcal{H})}(R)$ which is a subset of $\Gamma(\mathcal{P})$.

There is a homomorphism from $\mu: H \rightarrow \mathbb{Z}$ with kernel equal to the normal closure of $G$. We say an element $h \in H$ (ie a vertex of $\Gamma_{(H, \mathcal{H})}$ ) is in level $n$ if $\mu(h)=n$. If $C$ is a compact subcomplex of $\Gamma$ we say $C$ is in levels $L(C)$ through $M(C)$ if $L$ is maximal and $M$ is minimal such that for each vertex $v$ of $C, L \leq \mu(v) \leq M$. If $e$ is a $\mathcal{G}$-edge of $\Gamma_{(H, \mathcal{H})}(R)$ in level $K$ and $e$ has label $g \in \mathcal{G}^{ \pm 1}$ then the relation $t^{-1} g t=\phi(g)$ defines a $2-$ cell of $\Gamma_{(H, \mathcal{H})}(R)$ that slides $e$ to a path in level $K+1$. The path in level $K+1$ can be slid to level $K+2$ and so $e$ can be slid to any level above level $K$. 


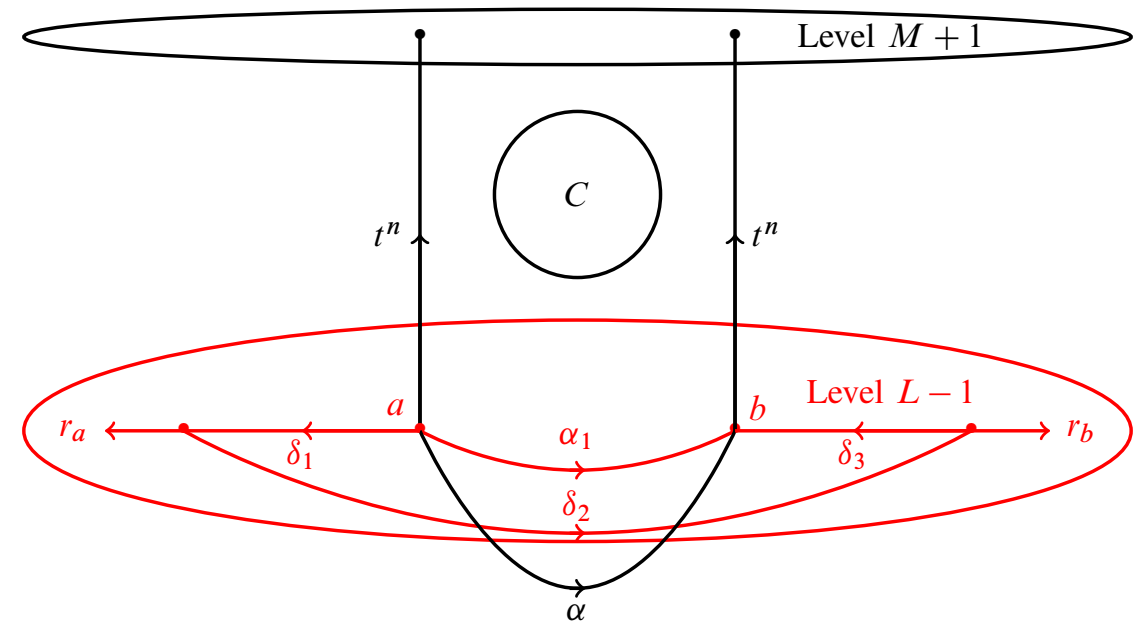

Figure 5: Sliding $\mathcal{G}$-edges of $\gamma$ up in any level $L-1$ through $M$ to level $M+1$ by a homotopy avoiding $C$.

The basic idea is to show that there is a compact set $D$ in $\Gamma_{(H, \mathcal{H})}(R)$ such that any loop in $\Gamma_{(H, \mathcal{H})}(R)-D$ is homotopic to a loop that can be slid to a single level (usually above the levels of $C$ ), by a homotopy avoiding $C$. Any path in a single level belongs to a translate of $\Gamma_{(G, \mathcal{G})}\left(R_{1}\right)$. The semistability of $G$ is used to move this loop (by a homotopy in that copy of $\left.\Gamma_{(G, \mathcal{G})}\left(R_{1}\right)\right)$ to a loop far from $C$. The simple connectivity at $\infty$ of $G$ shows this last loop is homotopically trivial in $\Gamma(\mathcal{P})-C$ and so $H$ is simply connected at $\infty$ in $Q$.

Let $D(C)$ be a compact subcomplex of $\Gamma_{(H, \mathcal{H})}(R)$ such that if $e$ is a $\mathcal{G}$-edge of $\Gamma_{(H, \mathcal{H})}(R)$ between levels $L(C)-1$ and $M(C)$ and the homotopy that slides $e$ to level $M+1$ intersects $C$, then $e$ is an edge of $D$. Also assume that if $X$ is a translate of $\Gamma_{(G, \mathcal{G})}$ in level $L-1$, then $D$ contains all bounded components of $X-D$ (there are only finitely many such $X$ that intersect $D$ ). Now let $\gamma$ be an edge path loop in $\Gamma_{(H, \mathcal{H})}(R)-D$. If no vertex of $\gamma$ is in level $L$ through $M$, then $\gamma$ can be slid up to be entirely in level $L-1$ or into some level above level $M$ by a homotopy avoiding $C$.

Otherwise, each $\mathcal{G}$-edge of $\gamma$ in a level $L-1$ through $M$ can be slid to level $M+1$ by a homotopy missing $C$. Call the resulting path $\gamma_{1}$ (so any edge of $\gamma_{1}$ between levels $L-1$ and $M$ is labeled $t$ ). Consider a maximal segment $\alpha$ of $\gamma_{1}$ with vertices all in levels $L-1$ and below. Then $\alpha$ is preceded (respectively followed) by a segment of $\gamma_{1}$ of the form $t^{-n}$ (respectively $t^{n}$ ) that begins (respectively ends) in level $M+1$. Let $a$ be the initial point of $\alpha$, and $b$ be the end point of $\alpha$; see Figure 5 .

Note that $a$ and $b$ are points of $\gamma$ and so do not belong to $D$. Hence there are proper $\mathcal{G}$-edge path rays $r_{a}$ and $r_{b}$ at $a$ and $b$ respectively that avoid $D$. Slide $\alpha$ (relative 
to $a$ and $b$ ) to level $L-1$ and call the resulting path $\alpha_{1}$. This homotopy is below the levels of $C$ and so avoids $C$. Now since $\Gamma_{(G, \mathcal{G})}\left(R_{1}\right)$ is semistable at $\infty$, there is a proper homotopy $A$ (in $\left.a \Gamma_{(G, \mathcal{G})}\left(R_{1}\right)\right)$ of $r_{a}$ to $\left(\alpha_{1}, r_{b}\right)$ relative to $a$. This homotopy gives an edge path of the form $\delta:=\left(\delta_{1}, \delta_{2}, \delta_{3}\right)$ that is homotopic to $\alpha_{1}$, where $\delta_{1}$ is an initial segment of $r_{a}$ (and so avoids $D$ ), $\delta_{3}^{-1}$ is an initial segment of $r_{b}$ (and so avoids $D$ ) and $\delta_{2}$ is a "far out" path in the image of $A$ (and so it also avoids $D$ ). By the definition of $D$ each edge of $\delta$ can be slid to level $M+1$ by a homotopy avoiding $C$.

This shows that $\gamma_{1}$ (and hence $\gamma$ ) is homotopic to a loop $\gamma_{2}$ in levels all above level $M$. Slide this loop up to a loop $\gamma_{3}$ in a single level (by a homotopy avoiding $C$, since $C$ is below level $M+1$ ). Note that $\gamma_{3}$ is a $\mathcal{G}$-path (since it has no $t$-edges). In any case, $\gamma$ is homotopic (in $\Gamma(\mathcal{P})-C$ ) to a $\mathcal{G}$-loop $\gamma_{3}$ in a translate, $v \Gamma_{(G, \mathcal{G})}\left(R_{1}\right)$, of $\Gamma_{(G, \mathcal{G})}\left(R_{1}\right)$ that avoids $C$. The simple connectivity at $\infty$ of $G$ implies that there is a compact set $E$ such that any loop in $v \Gamma_{(G, \mathcal{G})}\left(R_{1}\right)-E$, is homotopically trivial in $\Gamma(\mathcal{P})-C$. The semistability of $G$ implies $\gamma_{3}$ is homotopic in $v \Gamma_{(G, \mathcal{G})}\left(R_{1}\right)$ to a loop in $\Gamma_{(G, \mathcal{G})}\left(R_{1}\right)-E$ and so $\gamma_{3}$ and hence $\gamma$, is homotopically trivial in $\Gamma(\mathcal{P})-C$.

\section{References}

[1] S G Brick, ML Mihalik, The QSF property for groups and spaces, Math. Z. 220 (1995) 207-217 MR1355026

[2] G Conner, M Mihalik, Commensurated subgroups and ends of groups, J. Group Theory 16 (2013) 107-139 MR3008315

[3] G R Conner, M L Mihalik, Commensurated subgroups, semistability and simple connectivity at infinity, Algebr. Geom. Topol. 14 (2014) 3509-3532 MR3302969

[4] L Funar, D E Otera, On the wgsc and qsf tameness conditions for finitely presented groups, Groups Geom. Dyn. 4 (2010) 549-596 MR2653975

[5] R Geoghegan, Topological methods in group theory, Graduate Texts in Mathematics 243, Springer (2008) MR2365352

[6] R Geoghegan, M L Mihalik, Free abelian cohomology of groups and ends of universal covers, J. Pure Appl. Algebra 36 (1985) 123-137 MR787167

[7] R I Grigorchuk, On a problem of Day on nonelementary amenable groups in the class of finitely presented groups, Mat. Zametki 60 (1996) 774-775 MR1619929 In Russian; translation in Math. Notes 60 (1996) 580-582

[8] R I Grigorchuk, An example of a finitely presented amenable group that does not belong to the class EG, Mat. Sb. 189 (1998) 79-100 MR1616436 In Russian; translation in Sb. Math. 189 (1998) 75-95

[9] P de la Harpe, Topics in geometric group theory, University of Chicago Press (2000) MR1786869 
[10] G Higman, Subgroups of finitely presented groups, Proc. Roy. Soc. Ser. A 262 (1961) 455-475 MR0130286

[11] B Jackson, End invariants of group extensions, Topology 21 (1982) 71-81 MR630881

[12] F E A Johnson, Manifolds of homotopy type $K(\pi, 1), I$, Proc. Cambridge Philos. Soc. 70 (1971) 387-393 MR0290358

[13] F E A Johnson, On the first end invariant of an exact sequence, Mathematika 22 (1975) 60-70 MR0427483

[14] R Lee, F Raymond, Manifolds covered by Euclidean space, Topology 14 (1975) 49-57 MR0365581

[15] VM Lew, The semistability at infinity for multiple extension groups, PhD thesis, Cornell University, Ithaca, NY (1993) MR2689313 Available at http:// search.proquest.com/docview/304035762

[16] M L Mihalik, Semistability at the end of a group extension, Trans. Amer. Math. Soc. 277 (1983) 307-321 MR690054

[17] M L Mihalik, Ends of groups with the integers as quotient, J. Pure Appl. Algebra 35 (1985) 305-320 MR777262

[18] M L Mihalik, Ends of double extension groups, Topology 25 (1986) 45-53 MR836723

[19] M Mihalik, Semistability at $\infty$ of finitely generated groups, and solvable groups, Topology Appl. 24 (1986) 259-269 MR872498

[20] M L Mihalik, Semistability at infinity, simple connectivity at infinity and normal subgroups, Topology Appl. 72 (1996) 273-281 MR1406313

[21] J S Profio, Using subnormality to show the simple connectivity at infinity of a finitely presented group, Trans. Amer. Math. Soc. 320 (1990) 281-292 MR961627

[22] Y Shalom, G A Willis, Commensurated subgroups of arithmetic groups, totally disconnected groups and adelic rigidity, Geom. Funct. Anal. 23 (2013) 1631-1683 MR3102914

[23] J Stallings, The piecewise-linear structure of Euclidean space, Proc. Cambridge Philos. Soc. 58 (1962) 481-488 MR0149457

Department of Mathematics, Vanderbilt University

Nashville, TN 37240, United States

michael.1.mihalik@vanderbilt.edu

Received: 6 January 2016 Revised: 15 March 2016 